\title{
Review Article \\ Therapeutic Applications of Herbal Medicines for Cancer Patients
}

\author{
Shu-Yi Yin, Wen-Chi Wei, Feng-Yin Jian, and Ning-Sun Yang \\ Agricultural Biotechnology Research Center, Academia Sinica, No. 128, Section 2, Academia Road, Nankang, Taipei 115, Taiwan
}

Correspondence should be addressed to Ning-Sun Yang; nsyang@gate.sinica.edu.tw

Received 30 March 2013; Revised 17 June 2013; Accepted 19 June 2013

Academic Editor: Yoshiharu Motoo

Copyright (C) 2013 Shu-Yi Yin et al. This is an open access article distributed under the Creative Commons Attribution License, which permits unrestricted use, distribution, and reproduction in any medium, provided the original work is properly cited.

\begin{abstract}
Medicinal herbs and their derivative phytocompounds are being increasingly recognized as useful complementary treatments for cancer. A large volume of clinical studies have reported the beneficial effects of herbal medicines on the survival, immune modulation, and quality of life (QOL) of cancer patients, when these herbal medicines are used in combination with conventional therapeutics. Here, we briefly review some examples of clinical studies that investigated the use of herbal medicines for various cancers and the development of randomized controlled trials (RCTs) in this emerging research area. In addition, we also report recent studies on the biochemical and cellular mechanisms of herbal medicines in specific tumor microenvironments and the potential application of specific phytochemicals in cell-based cancer vaccine systems. This review should provide useful technological support for evidence-based application of herbal medicines in cancer therapy.
\end{abstract}

\section{Clinical Uses of Herbal Medicine with Anticancer Effects}

A range of clinical studies have indicated that a spectrum of anticancer activities from various herbal medicines can be detected. In this section, we have organized and classified the clinical use of a number of herbal medicines according to their suppressive effect on specific cancer types (Table 1).

1.1. For Breast Cancer. Although a specific role for vitamins and selenium in the prevention of breast cancer has not been established, some anticancer activities have been shown in vitro [1-3]. In a randomized controlled trial, 2972 patients with invasive or noninvasive breast carcinoma received either $200 \mathrm{mg}$ of vitamin A preparation (Fenretinide) per day or no therapy. At 97 months posttreatment there was a significant reduction in recurrence of local breast cancer in premenopausal women (HR: 0.65; 95\% CI: 0.46-0.92). However, no significant difference in metastasis or overall survival time could be demonstrated [4]. Interestingly, other studies have shown that long-term uptake of vitamin E may in fact have a negative effect on breast cancer patients [5, 6]. Currently, their rule seems to cause malabsorption or maldigestion in cancer patients suffering from a concomitant illness, moreover, providing patients adopt a balanced and healthy diet $[4,7]$.

Phytoestrogens are classified into water-soluble isoflavones and lipophilic lignans. Isoflavones are found in high abundance in soy beans, and lignans are found in linseeds wheat, fruit, flaxseeds, and vegetables $[8,9]$. Among six related clinical trials conducted so far, only one concluded that isoflavone was associated with a reduced risk of breast cancer [10]. Soy-derived phytoestrogens are popularly recommended for treating postmenopausal symptoms in women with breast cancer undergoing tamoxifen therapy. The principal constituents of soy bean plant extracts, including isoflavones genistein and daidzein, are structurally similar to $17 \beta$ estradiol and can confer weak estrogenic effects [11]. However, there is no evidence to support the recommendation of use of phytoestrogens either in treating breast cancer or for easing climacteric symptoms [12].

Investigations of traditional Chinese medicines (TCM) have uncovered a number of antibreast cancer agents, although most of their mechanisms of action have not yet been elucidated. These TCM herbs with antibreast cancer activities can be classified into six categories: alkaloids [13,14], coumarins [15, 16], flavonoids and polyphenols [17, 18], terpenoids [19], quinone [20], and artesunate [21] (Table 1). 
TABLE 1: Clinical use of herbal medicines exhibiting anticancer activities.

\begin{tabular}{|c|c|c|}
\hline Herbal medicine & Suppressive effects on carcinogenesis and cancer metastasis & References \\
\hline \multicolumn{3}{|c|}{ For breast cancer } \\
\hline Vitamin A (fenretinide) & $\begin{array}{l}200 \mathrm{mg} / \text { day significantly reduces the recurrence of local breast cancer in } \\
\text { premenopausal women }\end{array}$ & {$[4]$} \\
\hline Vitamin E & $\begin{array}{l}\text { Leads to malabsorption or maldigestion in cancer patients; balanced and } \\
\text { healthy diet }\end{array}$ & {$[5,6]$} \\
\hline Isoflavone & To reduce risk of breast cancer & {$[10]$} \\
\hline Isoflavones genistein and daidzein & To confer weak estrogenic effects & [11] \\
\hline Alkaloids & Inhibition of cancer cell growth & {$[13,14]$} \\
\hline Coumarins & Inhibition of cancer cell growth & {$[15,16]$} \\
\hline Flavonoids and polyphenols & Antiproliferation & {$[17,18]$} \\
\hline Terpenoids & MCF-7 cell apoptosis & {$[19]$} \\
\hline Quinone & $\begin{array}{l}\text { To induce G2-M arrest and autophagy by inhibiting the } \\
\text { AKT/mammalian target of rapamycin pathway in breast cancer cells }\end{array}$ & {$[20]$} \\
\hline Artemisunate & $\begin{array}{l}\text { Decrease the proliferation of human breast cancer cells from expressing } \\
\text { a high } \operatorname{ER} \alpha: \operatorname{ER} \beta \text { ratio }\end{array}$ & {$[21]$} \\
\hline \multicolumn{3}{|c|}{ For prostate cancer } \\
\hline Vitamins A-D and retinoid & Maintain homeostasis and prevent various metabolic disorders & {$[23]$} \\
\hline Vitamin E & Reduce the risk of lethal or advanced prostate cancer relative to nonusers & {$[30]$} \\
\hline Epigallocatechin-3-gallate (EGCG) & $\begin{array}{l}\text { Arrest LNCaP and DU145 prostate cancer cells at the G0-G1 phase of the } \\
\text { cell cycle }\end{array}$ & {$[34]$} \\
\hline & Inhibit metalloproteinase in vitro & {$[35]$} \\
\hline \multirow{2}{*}{ Soy isoflavones } & Inhibit $5 \alpha$-reductase activity & [37] \\
\hline & Chemopreventive activities & {$[22]$} \\
\hline Scutellaria baicalensis (baicalin) & Inhibit enzymatic synthesis of eicosanoids & {$[38]$} \\
\hline \multirow{4}{*}{ Baicalein } & $\begin{array}{l}\text { Impair the proliferation of androgen-independent PC-3 and DU145 } \\
\text { prostate cancer cells in culture }\end{array}$ & {$[39]$} \\
\hline & Induces cell-cycle arrest at the G0-G1 phase & [39] \\
\hline & $\begin{array}{l}\text { Induces apoptosis of prostate cancer cells at concentrations achievable in } \\
\text { humans }\end{array}$ & {$[40]$} \\
\hline & $\begin{array}{l}\text { Suppresses the expression of specific androgen receptor in prostate } \\
\text { cancer }\end{array}$ & {$[40]$} \\
\hline \multirow{3}{*}{ Lycopenes } & Decreases prostate cancer risk & [41] \\
\hline & Diminishes oxidative damage in lymphocytes & {$[42]$} \\
\hline & Significantly decreases levels of PSA and less oxidative damage & {$[42]$} \\
\hline \multirow[t]{2}{*}{ PC-SPES } & $\begin{array}{l}\text { Decreases serum testosterone concentrations }(P<0.05) \text {; decreases } \\
\text { serum concentrations of prostate-specific antigen }\end{array}$ & {$[43]$} \\
\hline & Antitumor efficacy against cancer cell lines & {$[44]$} \\
\hline Wedelia chinensis (Asteraceae) & Inhibits the androgen receptor (AR) signaling pathway & [47] \\
\hline \multicolumn{3}{|c|}{ For lung cancer } \\
\hline Platycodon grandiflorum (Campanulaceae) & Anticancer effect in lung cancer patients & \\
\hline Morus alba (Moraceae) & Anticancer effect in lung cancer patients & \\
\hline Prunus armeniaca (Rosaceae) & Anticancer effect in lung cancer patients & \\
\hline Rhus verniciflua (Anacardiaceae) & Anticancer effect in lung cancer patients & [49-51] \\
\hline Perilla frutescens (Labiatae) & Anticancer effect in lung cancer patients & \\
\hline Stemona japonica (Stemonaceae) & Anticancer effect in lung cancer patients & \\
\hline Tussilago farfara (Compositae) & Anticancer effect in lung cancer patients & \\
\hline Draba nemorosa (Brassicaceae) & Anticancer effect in lung cancer patients & \\
\hline
\end{tabular}


TABle 1: Continued.

\begin{tabular}{|c|c|c|}
\hline Herbal medicine & Suppressive effects on carcinogenesis and cancer metastasis & References \\
\hline \multicolumn{3}{|c|}{ For liver fibrosis and cancer } \\
\hline Inchin-ko-to (TJ-135) & \multirow{5}{*}{ Preventive effect on liver fibrosis } & {$[53]$} \\
\hline Yi Guan Jian (YGJ) & & {$[53]$} \\
\hline Yi Guan Jian (YGJ) & & {$[54]$} \\
\hline Fufang-Liu-Yue-Qing & & {$[55]$} \\
\hline Danggui Buxue Tang (DBT) & & {$[56]$} \\
\hline \multicolumn{3}{|l|}{ Salvianolic acid B } \\
\hline Curcumin & Suppressive effect on hepatic fibrogenesis and carcinogenesis & {$[57]$} \\
\hline \multicolumn{3}{|l|}{ Oxymatrine } \\
\hline Compound 861 & Suppressive effect on hepatic fibrogenesis & {$[58,59]$} \\
\hline Sho saiko-to (TJ-9) & Reduces/limits the progression of hepatocellular carcinoma & {$[60]$} \\
\hline \multicolumn{3}{|c|}{ For pancreatic cancer } \\
\hline $\begin{array}{l}\text { GDC-0449, IPI-926, XL-139 and } \\
\text { PF-04449913 }\end{array}$ & SMO antagonists; deregulation of sonic hedgehog homology (SHH) & {$[61]$} \\
\hline \multirow{3}{*}{ Cyclopamine } & $\begin{array}{l}\text { Inhibit SHH signaling by directly binding to the 7-helix bundle of the } \\
\text { SMO protein; arrest the growth of pancreatic tumors }\end{array}$ & {$[62]$} \\
\hline & $\begin{array}{l}\text { Weakens the recruitment of BMPCs into cancer cells and reduces the } \\
\text { formation of tumor vasculature }\end{array}$ & {$[63]$} \\
\hline & $\begin{array}{l}\text { The cancerous vascular system becomes unstable after treatment with } \\
\text { cyclopamine due to the expression of angiopoietin-1 }\end{array}$ & {$[63]$} \\
\hline
\end{tabular}

Some of these phytochemicals, such as curcumin and artemisinin, have well-known chemical structures. Compounds in these categories have been taken as health foods or dietary supplement for decades. However, evidence-based in vivo studies and clinical trials are still recommended for routine public use or specific clinical applications.

1.2. For Prostate Cancer. Prostate cancer is characterized by a long latency period, a strong dietary influence, and limited treatment strategies for the advanced disease; therefore, many patients turn to complementary and alternative medicine (CAM) with the belief that these medicines represent a viable therapeutic option that may be free of adverse side effects [22]. This folkloric belief, strongly upheld in many Asian cultures, needs to be substantiated with systematic, evidencebased research. Vitamins, including vitamins A-D and retinoids, are organic compounds that cannot be synthesized by humans and must be ingested to maintain homeostasis and prevent various metabolic disorders [23]. Emerging evidence indicates that inflammation might have a crucial role in the genesis of prostate carcinoma [24-26]. A number of clinical trials have sought to evaluate the anti-inflammatory activities of vitamins on prostate carcinogenesis [27]. Despite a lack of convincing evidence, vitamin and mineral supplements are used extensively by patients that are diagnosed with prostate cancers $[28,29]$. The belief is that such supplements might actually prevent or treat inflammation-associated disease and at the very least not cause harm [22]. Among smokers, daily ingestion of $>100$ IU of vitamin $\mathrm{E}$ was reported to produce a $56 \%$ reduction in risk in lethal or advanced prostate cancer relative to nonusers [30]. On the other hand, a selenium and vitamin E chemoprevention trial (SELECT) that aimed to determine whether vitamin $\mathrm{E}$ and/or selenium supplementation could reduce prostate carcinogenesis showed that dietary supplementation with vitamin E in fact statistically increased the risk of prostate cancer among healthy men [31, 32]. Together these clinical data suggest that the application of vitamin E may be specific for only treating inflammationassociated features in prostate cancer patients, rather than affecting anticancer activity. Future studies are needed to address such an apparent contradiction between effects in healthy and cancer populations.

The use of medicinal herbs and their derivative herbal extracts that contain numerous polyphenolic compounds may contribute to the lower incidence of prostate cancer in Asian populations relative to Caucasians and African Americans [33]. Many polyphenols (e.g., isoflavones) are phytoestrogens that can bind to estrogen receptors and elicit estrogenic effects in target tissues or organs. Some specific compounds, in particular the four active polyphenolic compounds in green tea, epicatechin, epigallocatechin, epicatechin-3-gallate, and epigallocatechin-3-gallate (EGCG), the soy isoflavones, as well as Scutellaria baicalensis, $\beta$-carotene, and the lycopenes have all been studied for their effect on prostate carcinoma [22]. Using specific bioassays, EGCG in green tea was demonstrated to arrest LNCaP and DU145 prostate cancer cells at the G0-G1 phase of the cell cycle [34] and inhibit metalloproteinase in vitro, although the effect was achieved at a much higher concentration than the serum levels detected in humans who consumed moderate amounts of green tea [35]. In a Phase II study of green tea in the treatment of patients with androgen-independent metastatic prostate carcinoma, one patient achieved a prostate-specific antigen (PSA) response of $>50 \%$ that lasted 
for approximately one month. Patients, however, suffered marked symptoms of toxicity in this study, most notably diarrhea, nausea, and fatigue. Investigators concluded that green tea has limited antineoplastic activity, as defined by a decline in PSA levels, against androgen-independent prostate cancers [36]. For soy isoflavones, they have been shown to inhibit $5 \alpha$-reductase activity, the enzyme that is functionally responsible for the conversion of testosterone to the more potent androgen dihydrotestosterone [37]. Several preclinical randomized studies have also evaluated the potential therapeutic effects of soy isoflavones as chemopreventive agents [22]. For Scutellaria baicalensis, it contains very high levels of baicalin, a flavone glycoside that inhibits enzymatic synthesis of eicosanoids, which are important mediators of inflammation and prostate tumor cell proliferation [38]. Baicalein (a flavone) impairs the proliferation of androgenindependent PC-3 and DU145 prostate cancer cells in culture and induces cell-cycle arrest at the G0-G1 phase [39] and apoptosis at concentrations achievable in humans [40]. Baicalein drastically suppressed the expression of specific androgen receptor in prostate cancer at clinically achievable concentrations [40]. For lycopenes, although most studies have used mixtures of tomato products, the association of decreased prostate cancer risk and tomato product intake has led to the specific focus on the use and effect of lycopene [41]. Chen and colleagues studied the effect of lycopene levels and measures of oxidative damage in patients undergoing prostatectomy for localized prostate cancer [42]. They found that oxidative damage in lymphocytes from these patients was diminished after dietary intervention compared with pretreatment levels and that the prostate tissues in treated patients revealed significantly decreased levels of PSA and less oxidative damage. Since it still remains uncertain whether lycopene itself causes the effect, or whether a more complex food extract is responsible, additional randomized trials are needed to assess the efficacy of lycopene in chemoprevention activities.

Sales of the herbal extract PC-SPES as a dietary supplement for "prostate health" began in the mid-1990s. The name PC-SPES is derived from PC for "prostate cancer" and "spes," the Latin word for hope. This botanical mixture is used primarily for treatment of prostate carcinoma [43]. The formulation contains extracts of eight herbs, Ganoderma lucidum, Scutellaria baicalensis, Rabdosia rubescens, Isatis indigotica, Dendranthema morifolium, Serenoa repens, Panax notoginseng, and Glycyrrhiza uralensis, that were selected based on either their use in Chinese medicinal therapy for urinary problems or their antitumor efficacy against cancer cell lines [44]. In addition, a series of clinical studies have described the effects and mechanisms of PC-SPES activity [45]. Although the therapeutic application of PC-SPES seemed to be promising, unfortunately, PC-SPES was recalled and withdrawn from the market because certain batches of testing PC-SPES samples were found to be contaminated with US Food and Drug Administration-(FDA-)controlled prescription drugs. To our knowledge, the FDA has not approved so far the use of PC-SPES in cancer treatment. More evidence and correlative information to demonstrate the in vitro and clinical efficacy of this herbal mixture are needed.
Lin et al. [46] showed that Wedelia chinensis (Asteraceae), an oriental medicinal herb containing various compounds such as indole-3-carboxylaldehyde, wedelolactone, luteolin, and apigenin, is capable of suppressing androgen activity. Moreover, oral administration of $W$. chinensis extract impeded prostate cancer tumorigenesis. This anticancer action of $W$. chinensis extract was subsequently demonstrated to be due to three active compounds that can inhibit the androgen receptor (AR) signaling pathway [47]. Recently, our own study showed that a different set of phytochemicals extracted from $W$. chinensis plants can confer potent and specific anti-inflammatory bioactivities in vitro and in vivo. These activities resulted in strong anticolitis activities in test mice [48]. Future studies of $W$. chinensis for chemoprevention or as a complementary medicine against prostate cancer in humans are warranted.

1.3. For Lung Cancer. Lung cancer is one of the most deadly cancers, and the lung is a common site of metastasis of tumors from other tissues in the body. Standard chemotherapy regimes often have limited survival benefits due to the severe toxicity $[49,50]$ of the various anticancer agents, such as gemcitabine, paclitaxel, docetaxel, etoposide, and vinorelbine. Recent reports have suggested that herbal medicines and their phytochemicals which seem to have lower or little toxicity may provide an attractive strategy for lung cancer therapy. Traditionally, herbal plants such as Platycodon grandiflorum (Campanulaceae), Morus alba (Moraceae), Prunus armeniaca (Rosaceae), Rhus verniciflua (Anacardiaceae), Perilla frutescens (Labiatae), Stemona japonica (Stemonaceae), Tussilago farfara (Compositae), and Draba nemorosa (Brassicaceae) have been used to treat lung cancer [51]. Clinically, the proportion of patients that use herbal medicines as adjuvants alongside conventional (e.g., chemotherapy) treatment for lung cancer is as high as $77 \%$ [52]. Herbs are mainly used in lung cancer to reduce therapy-associated toxicity and cancerrelated symptoms and sometimes to directly increase anticancer effects [4]. However, it is important to note that some CAM methods or remedies may have adverse effects or reduce the efficacy of conventional treatment, and the primary justification for use of traditional herbal medicines remains empirical evidence, case studies, and hypothetical physiological effects [4].

1.4. For Liver Fibrosis and Cancer. Liver fibrogenesis is a gradual process of increased secretion and decreased degradation of extracellular materials, which can be initiated by activation of hepatic stellate cells (HSCs) [64, 65]. The number of deaths due to hepatocellular carcinoma (HCC) has steadily increased over the last decade. Unfortunately, there are no successful, clinically satisfactory therapies for patients suffering HCC. Herbal medicines are being considered as one possible strategy against liver fibrosis and HCC. Three medicinal herbs are already used as official drugs in China, Japan, and other parts of Asia. Different chemically induced fibrosis models were designed using the rat liver system to assess the preventive effects of specific herbal extracts on liver fibrosis. Formulations assessed include Inchin-ko-to (TJ-135) [53], Yi 
Guan Jian (YGJ) [53], Yi Guan Jian (YGJ) [54], Fufang-LiuYue-Qing [55], and Danggui Buxue Tang (DBT) [56]. In 2007, Luk et al. [57] provided a systematic review of the mechanisms of action of medicinal herbal compounds, such as salvianolic acid $B(S A B)$, oxymatrine, and curcumin in the treatment of hepatic fibrogenesis and carcinogenesis. Although some of these herbal medicines, such as YGJ, are traditionally used to treat human liver fibrosis, the therapeutic or clinical anticancer effect of these herbal mixtures in liver tissue has not been fully elucidated. The further identification of as yet unknown effective components in these herbal extract is critical for their pharmacological use and improvement.

A combination of 10 herbs (named compound 861), including Salvia miltiorrhiza (sage), Astragalus membranaceus, and Spatholobus suberectus known in TCM as the "king herb" components of the formula, that is, the herbs that are pharmacologically active, and seven others (modifiers of toxicity that act synergistically with the king herbs to improve immune function), has been tested in a number of experimental studies for antifibrotic properties. Two uncontrolled open trials of 60 and 22 patients with chronic hepatitis B who were treated with compound 861 reported a beneficial effects on liver fibrosis, with the majority of treated patients showing both clinical and histological improvement $[58,59]$. Since these clinical studies of compound 861 did not satisfy quality control criteria, clinicians consider that additional well-designed trials are needed for routine and authorized clinical use of compound 861 for the treatment of hepatitis B-induced liver fibrosis.

A large number of clinical reports have indicated the therapeutic effects of one Japanese traditional medicine (kampo yaku) Sho saiko-to (TJ-9). This medicine is a combination of seven herbs traditionally used for treating liver diseases $[66,67]$. However, little clinical data on the efficacy of TJ9 in preventing human liver cancer has been reported. In a long-term (5 years) randomized controlled study, patients that were positive for hepatitis B surface antigen ( $\mathrm{HBsAg}$ ) received a dose of $7.5 \mathrm{~g} /$ day aqueous $\mathrm{TJ}-9$ extract together with the standard treatment using interferon. Upon followup, the cumulative development of hepatocellular carcinoma (HCC) was found to be significantly lower than that of the controls (i.e., patients without TJ-9 treatment) [60]. Unfortunately, TJ-9 is contraindicated for patients with hepatic cirrhosis or acute respiratory failure in Japan, because some of these patients were found to contract interstitial pneumonia after drug administration [68]. Therefore, well-designed future trials that can address the specificity of TJ-9 or its major active components in inhibition or suppression of the progression of viral hepatitis-induced hepatocellular carcinoma or metabolic liver cancers are needed [69].

1.5. For Pancreatic Cancer. Smoothened (SMO), a component of the sonic hedgehog homology ( $\mathrm{SHH}$ ) signaling pathway, has been shown to play a key role in the cellular behavior of cancer stem cells [70]. The deregulation of SHH was considered as an important factor that can drive or maintain the progression of pancreatic cancer [71]. There are some SMO antagonists that, such as GDC-0449, IPI-926, XL-139, and
PF-04449913, are being evaluated with high hope for treatment of pancreatic cancers [61]. Cyclopamine, a steroidal alkaloid extracted from Veratrum californicum, can efficiently inhibit SHH signaling by directly binding to the 7-helix bundle of the SMO protein. This complex can further impact upon the function of 12-transmembrane receptor patched-1 (PTCH-1) and thereby influence the structure of SMO [62]. It needs to be noted here that cyclopamine not only can weaken the recruitment of bone marrow precursor cells (BMPCs) into cancer cells, but also can reduce the formation of tumor vasculature [63]. The cancerous vascular system becomes unstable after treatment with cyclopamine due to the expression of angiopoietin-1, an angiogenic factor found in the tumor microenvironment, which is under the regulation of SHH. Cyclopamine has been explored as an SMO activity suppression agent and to arrest the growth of pancreatic tumors [63]. Encouraging findings suggest that this phytochemical obtained from a traditionally used TCM herb should be systematically explored in the future for efficacious SMO-targeting anticancer drugs.

\section{Use of Herbal Supplements as Adjuvants in Conventional AntiCancer Therapies}

Numerous Chinese herbal medicines are being used in combination with chemotherapy or radiotherapy to improve the efficacy of cancer therapy and reduce side effects and complications (Figure 1), although this practice is highly frowned upon by many western physicians. Understanding of the use of specific herbal medicines as adjuvants to conventional therapy, therefore, needs to be increased in consultation and coordination with physicians and other health care providers. This section outlines evidence for use of herbal medicines as adjuvants to conventional drug-based, chemo- or radiotherapy regimes in cancer treatment. On the other hand, this section also summarizes the challenges or limitations for clinical use of these herbal medicines.

\subsection{Common Use of Herbal Medicines as Adjuvant Treatment} in Chemo- or Radio-Cancer Therapy. For the above adjuvant anticancer therapy studies, herbal medication in general was applied as a combination therapy with the conventional chemotherapy to hopefully increase the therapeutic benefit and quality of life (QoL) as well as to decrease the side effects or complications. Between $28 \%$ and $98 \%$ of ethnic Chinese cancer patients in Asia [72-74] and 25\% to $47 \%$ of those living in North America are reported to have used herbal medicines as part of their cancer care $[75,76]$. Although a number of herbal medicines have been found to be adjunctive in chemoand radiotherapy, most clinical trials or studies have been reported mainly, if not only, in China or other Asian countries and they are virtually not cited on PubMed. In 2010, Qi and colleagues [77] provided a systematic review of Chinese herbal medicines in clinical trials, mainly as adjuvant treatments to reduce complications and side effects of chemo- or radiotherapy. Several traditionally used Chinese herbal medicines, including astragalus $[78,79]$, Turmeric (curcumin) 


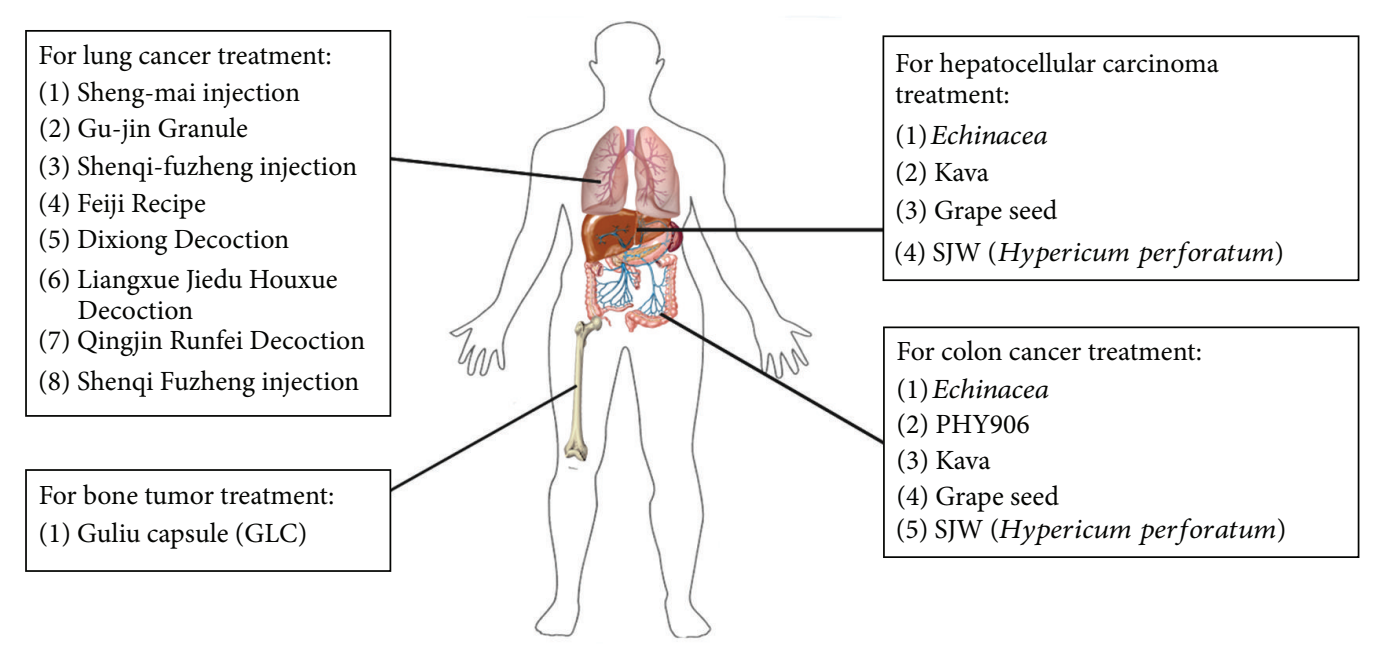

FIGURE 1: Medicinal herb “extracts” or “formulations” being tested as adjuvant treatments for chemo- or radiotherapies against various cancers.

[80-82], Ginseng [83-85], TJ-41 (Bu-Zhong-Yi-Qi-Tang) $[86,87]$, PHY906 [74, 88-90], Huachansu [91, 92], and Kanglaite $[93,94]$, are commonly used by cancer patients to either "treat" cancer and/or "reduce the toxicity" induced by chemotherapy or radiotherapy. Preclinical and clinical studies have indicated that these herbal medicines may possess a number of advantages in terms of suppression of tumor progression, by increasing the sensitivity of chemo- and radiotherapeutics, improving immune system function, and easing the tissue/physiology damage caused by chemo- and radiotherapeutics. However, most studies to date are empirical (i.e., not well controlled) clinical studies or observations that mainly report reduction in side effects and complications during or after chemotherapy and radiotherapy. Some traditional herbal formulations, including Bojungikki-tang [87], Kang-Fu-Zhi-Tong [95], PHY906 [88], Xiao-Chai-Hu-Tang, Huang-Lian-Jie-Du-Tang, and Yin-Chen-Wu-Ling-San [96], have been observed, "detected," or "claimed" to protect liver function, reduce cancer-related fatigue and pain, improve respiratory tract infections and gastrointestinal side effects, and even ameliorate the symptoms of cachexia. Often, these clinical results do not meet the standard US FDA requirements for clinical trials, but they may still offer some insight into traditionally used herbal medicines as adjuvant treatments for cancers. They may also provide useful pointers for the development of future botanical drugs as cancer primary or adjuvant therapies $[74,77,97]$.

2.2. For Lung Cancer. In a randomized controlled trial (RCT) with 63 patients with non-small-cell lung cancer (NSCLC), Sheng-mai Injection (Yảan Sanjiu Pharmaceutical Co., China) and Gu-jin Granule (Jiangyin Tianjiang Pharmaceutical Co., China) were observed to enhance median survival time $(P=0.014)$ and response rate increase to $48.5 \%(16 / 33)$, compared to the untreated control $(32.2 \%=9 / 28)$ in the control group $(P=0.0373)$, while all test groups were treated with a combination of navelbine and cisplatin (NP) chemotherapy [98]. In another clinical trial with Shenqifuzheng injection (Lizhu Co., China) among 232 NSCLC patients enrolled, herbal injection significantly improved the response rate and QoL of lung cancer patients, evaluated by using the QoL scale of European Organization for Research on Treatment of Cancer (QLQ-C30) [99]. Furthermore, the randomized controlled trial for Feiji Recipe treatment was also observed to enhance the clinical therapeutic efficacy and alleviate side effects of chemotherapy [100], as shown with an increase on scores in role, social, and economic status $(P<0.05$ or $P<0.01)$, again based on QLQ-C30 questionnaire [101]. Recently, Xu et al. [102] applied a high quality of clinical trial methodology to examine the effect of TCM on improving QoL of postoperative non-small-cell lung cancer (NSCLC) patients. They clearly presented the design and protocol for a placebo-controlled, double-blinded RCT and were able to systematically provide evidence for the effectiveness of chemotherapy combined with TCM in improving QOL of postoperative NSCLC patients. The result is expected to provide support for integrative optimization of "combined" treatment of lung cancer patients.

One of the major risks of conventional treatment in lung cancer patients is radiation pneumonitis, caused by radiotherapeutic intervention [103]. Increasing evidence has been reported on the beneficial efficacy of certain herbal medicines such as Dixiong Decoction [104], Liangxue Jiedu Huoxue Decoction [105], Qingjin Runfei Decoction [106], and Shenqi Fuzheng injection [107] (Figure 1). These herbal formulations were reported to significantly lower the incidence of radiation pneumonitis and improve clinical radiographic physiologic (CRP) dyspnea score and the Radiation Therapy Oncology Group (RTOG) grading score, in groups of NSCLC patients undergoing radiotherapy treatment. These findings also revealed some of the possible adverse effects and potential uses of specific herbal medicines in combinational therapy alongside conventional chemotherapy. The broad range and heterogeneity of herbal medicine intervention and the resultant effects still pose a challenge to high-powered analysis of specific herbal medicines and their applications for evidence-based use in cancer therapies. Therefore, high level quality control to ensure consistent batch preparation and 
systematic pharmacokinetic studies are required for all test herbal medicines and their activity against lung cancer [108], not only in human studies, but also in experimental animal systems to support evidence-based application.

2.3. For Colon Cancer. In oncology, drug interactions are important because of the narrow therapeutic index and inherent toxicity of many anticancer agents [109]. Previous studies indicated that the activity of cytochrome P450 enzymes (CYP enzymes) in the gastrointestinal wall is one of the most important factors that can alter the bioavailability of orally administered anticancer agents that are substrates of CYP3A [110]. A number of herbal supplements including Echinacea, kava, grape seed, and St John's wort (Hypericum perforatum) are also considered to be inducers of CYP [111] (Figure 1). Because of the increased use of herbal products by cancer patients, more consideration needs to be given to their combined use with anticancer agents $[109,112]$. The administration of St. John's wort was shown to induce intestinal and hepatic expression of CYP3A [113] and be beneficial for the metabolism of irinotecan [114], a camptothecin derivative that can result in DNA damage on interaction with topoisomerase I. St. John's wort is hence used empirically in the treatment of metastatic carcinoma of the colon or rectum.

Recent studies based on epidemiological modeling have demonstrated interesting patterns suggesting that herbal treatment may improve prognosis in advanced colon cancer patients when used as an adjuvant therapy $[115,116]$. The therapeutic mechanisms of traditional Chinese medicine in metastatic cancer have been discussed in terms of a hypothetical, dualistic antiproliferation and immune-stimulation model of tumor progression and regression [117]. Clinically, between $30 \%$ and $75 \%$ of patients with colon cancer are estimated to use CAM, but systematic or statistical evidence of survival efficacy is still limited. In one study with a 10-year followup of colon cancer patients $(n=193)$ who presented to a San Francisco Bay Area Center for Chinese medicine, authors compared the survival rate in patients choosing a short-term treatment regime lasting for the duration of their chemotherapy/radiotherapy period with those choosing a continuing long-term treatment. They also compared the survival of patients treated with Pan-Asian medicine plus vitamins $(\mathrm{PAM}+\mathrm{V})$ with that of concurrent external controls from the Kaiser Permanente Northern California and California Cancer Registries [118]. In this study, some modern methods, including Kaplan-Meier and traditional Cox regression, were used for analyses of causal inference, namely, propensity score and marginal structural models (MSMs), which have not been previously used in studies of cancer survival in response to treatment with Chinese herbal medicine. Results indicated that PAM+V combined with conventional therapy, as compared with conventional therapy alone, reduced the risk of patient death at stage I by $95 \%$, stage II by $64 \%$, stage III by $29 \%$, and stage IV by $75 \%$. No significant difference was observed between short-term versus long-term PAM+V administration [118]. This was apparently a sound clinical investigation and suggests that prospective trials combining $\mathrm{PAM}+\mathrm{V}$ with conventional chemotherapy/radiotherapy may be clinically justifiable in future systematic studies.
Accumulating clinical studies show that some TCM preparations, including Pi-Sheng Decoction and Yi-Qi-ZhuYu Decoction, may be useful in reducing side effects and enhancing the drug effect of chemotherapy for colorectal cancer [119]. For preventing recurrence and metastasis, JianPi-Xiao-Liu Decoction, Fu-Zheng Capsule, and Qu-Xie Capsule were used to decrease the recurrence and metastasis of colorectal cancer in a subsequent consolidation therapy after radical resection of patient's tumor. For improving the quality of life, Jian-Qi-Jie-Du Decoction, Jian-Pi-Yi-Qi Decoction, Fu-Pi-Yi-Wei Decoction, and Ai-Di injection were reported to enhance the antitumor "curative" effect of chemotherapy, reduce the side effects of chemotherapy, improve the immune function, and extend survival time in colorectal cancer patients. However, with the advancement of colorectal cancer treatment model, TCM theories and clinical studies on the typing of syndrome differentiation apparently are still lagging behind. In addition, current studies often have not addressed the issues on the anticancer properties or the observed beneficial health maintain/survival effects of treated TCMs. It is not only desirable, but also in fact necessary to further study the action model and the associated biochemical and physiological mechanisms of these anticancer mode herbs, as a milestone for future TCM research [119].

2.4. For Hepatocellular Carcinoma. The traditional Chinese medicine term or pathological classification of unresectable hepatocellular carcinoma (UHCC) is "liver stasis" [120]. Many clinical studies from China have indicated that TCM, such as Shentao Ruangan pills and hydroxycamptothecin, plus chemotherapy can significantly alleviate the symptoms, enhance therapy tolerance, stabilize tumor size and augment immunological function, reduce the incidence rate of adverse events, and prolong survival time of UHCC patients [121-123]. Although these studies may be criticized individually for lacking quality at the international level, together they do seem to suggest that the administration of TCM may warrant additional trials for patients with UHCC. Future clinical trials with TCM for UHCC need to have sufficient methodological quality and should be pursued in accordance with the Consolidated Standards of Reporting Trials (CONSORT) statement (see Section 3). In particular, rigorously designed, multicenter, large, randomized, double-blind, controlled trials are necessary [124].

2.5. For Other Cancers. Over the past two decades, a number of Chinese herbal medicines have been noted for their radiosensitization and radioprotection effects during radiotherapy of cancers, including bone cancer as well as head and neck tumors [125]. Cho and Chen [126] reported that a combination of TCM with radiotherapy not only enhanced therapeutic outcomes, but also improved the performance status of patients with nasopharyngeal cancer. Su and colleagues [127] consistently found that Guliu capsules (GLC) combined with $\mathrm{Sr}-89$ conferred therapeutic effects in the treatment of metastatic bone tumors. They found that combined GLC and Sr-89 treatment was effective against metastatic bone tumor and improved patients' QOF enhancing ostalgia relief rate 
and decreasing hemotoxicity. In brain tumor treatment, Quan and colleagues [128] also reported that TCM, in combination with radio- or chemotherapy, had an effect on tumor growth inhibition, survival time, and QOL enhancement in brain tumor metastases. These findings further indicate the potential application of TCM in the therapy of different cancers (Figure 1).

\subsection{Challenges for the Use of Herbal Medicine in Cancer} Therapy. Although traditional herbal medicines, phytomedicines, medicinal foods, and complementary or alternative medicines have been increasingly used over the past decade in European and North American countries, they seem to have not generated interest or been accepted by mainstream medicine practitioners in western countries, especially in standard care for cancer patients. The key issue considered by many biomedical scientists has been the lack of evidencebased information/guidelines for routine and regulatory application of herbal medicines as "drugs" for use in public health. The sticking points hindering the use of phytomedicines can be attributed to six major issues: (1) lack of consistent and reliable sources of authentic medicinal plant materials, with respect to species verification and authentication, cultivation using good agricultural practice protocols, and standardized/normalized methods and technology for plant extraction/mixture preparation; (2) lack of definitions and routine preparation of the biochemical/biological ingredients and compositions of herbal medicines or the phytochemicals/phytocompounds derived from medicinal plants, with respect to identification of metabolite profiles, index compounds, and putative active compounds or metabolites; (3) general and specific safety considerations, including tolerable high dosage, minimal effective dosage, and specific usage; (4) proof of efficacy in treating or assisting specific cancer patients, including lack of results/data from preclinical animal studies, execution of bona fide, and double-blind, placebo-included, statistician-assisted clinical trial studies; (5) highly complex "personalized" prescriptions or formulations for the use of some traditional medicines (e.g., in TCM) that may be mystified by a "secret ingredient" in specific formulations; and (6) the criminal act of supplementation/ "spiking" highly potent western chemical drugs into herbal medicines in counterfeit activities. Without addressing all of the above issues, we cannot meet the challenges of modernizing herbal medicines. Although we have reviewed a spectrum of laboratory, preclinical and clinical studies on potential applications of herbal medicines for cancer patients' care in an inclusive fashion, a great many of these studies did not follow the stringent requirements, procedures, and protocol needed for developing western style drug or medicinal foods. Systematic and correlated efforts among researchers of our scientific communities are therefore urgently needed.

It is also important to note that the central tenet in recent western medicine is that a drug should be composed of wellknown chemical components or a pure single compound that selectively interacts with known and specific molecular target(s) in our body system. However, the search for single molecules that can modify single or highly specific key factors in a disease process is now recognized as a difficult and sometimes inappropriate strategy, because a large volume of studies on genomics, proteomics, and metabolomics studies have shown that many clinically used commercial drugs (e.g., aspirin, doxorubicin, etc.) may in fact bind and work on multiple molecular targets. Multiple cell types, target molecules, and/or multiple signaling pathways are known to contribute to various diseases. Herbal extracts/mixtures prepared as traditional phytomedicines represent a combinational chemistry and "thus claimed" to encompass a vast and useful repertoire of chemical entities that can confer a complex and yet integrated effect on a spectrum of molecular and cellular components and functions, resulting in a profound and balanced medicinal activity. Unfortunately, according to the current FDA and $\mathrm{NIH}$ cancer clinical trial regulations in USA, such "claims" often directly conflict with the present guidelines or guidance. One major drawback in the integration of herbal medicines into mainstream western medicines is, therefore, the lack of defined molecular targets. With regard to this concern, recent research findings revealed from a spectrum of omics studies strongly suggest that a multifactorial mode of action and multitarget pharmaceutical activity may in fact already be the "norm" for a spectrum of currently used clinical drugs. As a result, there may be much less difference in terms of the complexity of molecular targets aimed by single compound drugs versus complex herbal medicine extracts than was originally assumed, as we previously demonstrated in a cancer cell line study [129]. We may then further project that the "multi-target" approach or activity believed for various herbal medicines may in fact be "reasonable and understandable" and therefore be positively considered and prepared in botanical drug development. Pooling data from individual trials by using a meta-analysis approach may be a useful strategy to interpret at the results of a group of inconclusive trials [130].

The uncertain or not well-defined composition of herbal products also raises questions about their safety, such as the evidence indicating that some herbal extracts may have harmful interactions with specific prescription drugs [131]. To address this issue, the establishment of optimized CMC (chemistry, manufacture, and control) conditions for each herbal preparation will need to be considered as important technology for confirming and standardizing the composition of specific medicinal herb components. Toward this aim, we believe that the pattern-oriented approach (fingerprint analysis) is a good strategy, because it can evaluate the integrative and holistic properties of test herbal medicines by comparing the similarities, differences, and correlation of the results from analyses of the whole production process, including manufacture, processing and storage of raw materials for preparation, intermediate products, finished products, and the distribution products [132, 133]. Yongyu and colleagues [134] have systematically reviewed fingerprint methods for analyses of herbal medicines. The fingerprint profiling of therapeutically used herbal medicines can be employed as a reference or index for quality control of phytochemical composition, and the results can be used in future clinical applications. Furthermore, the fingerprinting profiles can also be coordinated with and employed for therapeutic 
efficiency. This study approach was recently evaluated by an investigation of randomized controlled clinical trials (see Section 3).

In order to treat specific diseases, it is desirable that "modern drugs" can be generally applied to most patients with the same disease, although personalized medicine is becoming more popular. In traditional herbal medicines, mixtures of herbal extracts, comprising multiple phytocompounds, presumably regulating multiple targets for two or three medical indications are often used in a prescription. A major challenge for clinical use of such herbal remedies in cancer therapeutics is the evaluation of "true" active components and their targets for such multiple indications. Although modern chemical drugs and conventional herbal medicines may seem to be very different, they may, in fact, share some pharmacological foundations. As some herbal medicine classes have a common structural scaffold, this similarity may account for their potency in similar target groups [135]. It is believed that these structures and the activity/function information will become one of the most important indices for medicinal chemists to efficiently classify and seek specific pharmaceutical activities and for effectively optimizing herbal chemicals.

\section{Evidence of the Effect of Herbal Medicines in Randomized Controlled Trials}

Randomized controlled trials (RCTs) (or randomized comparative trials) currently serve as the gold standard for most clinical trials and provide the best evidence of the efficacy of healthcare interventions [136]. Carefully planned and wellexecuted RCTs often obtain the best estimates of treatment effect and thus help guide clinical decision making; therefore, considerable effort is put into improving the design and reporting of RCTs [137, 138]. Linde et al. [139] commented that reporting quality may vary across different types of complementary therapies, with herbal medicine trials being apparently superior to homeopathy and acupuncture trials. Also, reporting quality differed among different individual botanical medicines and improved continuously for decades from the 1980s to the 2000s [140]. With these controversies, in June 2004 an international group of pharmacologists, methodologists, pharmacognosists, and trialists met for a consensus-making meeting, which then led to the development of recommendations for the reporting of herbal medicine trials in Toronto, Canada [141]. An elaboration of CONSORT statement was put forward that aimed to aid researchers to more accurately assess the internal/external validity and reproducibility of herbal medicine trials, to allow a more accurate assessment of safety and efficacy of herbal medicines [141, 142]. Among the 22 CONSORT checklist items, 9 of them were elaborated to enhance their relevance to the trials of herbal interventions, including the detailed recommendations for 1 item (item 4 (interventions)) and minor recommendations for 8 items (item 1 (title and abstract), item 2 (background), item 3 (participants), item 6 (outcomes), item 15 (baseline data), item 20 (interpretation), item 21 (generalizability), and item 22 (overall evidence)) [141]. Specifically, the detailed recommendation in item 4 addressed the concerns of the herbal medicine intervention, which is still need extensive elaboration. These recommendations have been developed to improve the reporting of RCTs.

Although TCM and other herbal medicines are being used worldwide, their efficacies have only been studied in a sporadic way, with very few properly randomized and controlled studies. Trials of note that have employed a high standard of clinical trial methodology include Mok and colleagues [143] and Chan and colleagues [144]. Mok and colleagues examined the possible role of Chinese herbal medicine in reducing chemotherapy-induced toxicity. They reported that traditional Chinese herbal medicine seemed to have a significant effect on the control of nausea in patients with early-stage breast or colon cancers, but these herbal medicines did not reduce the hematologic toxicity associated with chemotherapy. In addition, Chan et al. [144] conducted a randomized, placebo-controlled trial to evaluate the efficacy of test TCMs in improving QOL and reducing chemotoxicity and possible decrease in the side effects of systemic chemotherapy and the immune system status of patients undergoing a standard treatment for ovarian cancers. In this study, ovarian cancer patients were randomized to receive either the test TCM formulation or a placebo in addition to standard chemotherapy. The primary outcome was recorded by the global health status (GHS) score and assessed by the European Organization for Research and Treatment of Cancer questionnaire, and the secondary outcomes were examined using other QOL items, chemotoxicity levels defined according to the World Health Organization (WHO) criteria, and alterations in specific immune functions. The results suggest that TCM exerted effects in maintaining immune function (e.g., lymphocyte count and cytokine activities) rather than improving QOL. However, as these randomized trials failed to recruit sufficient study numbers, we may need to conclude that, in order to fully evaluate and demonstrate specific bioactivities and the merits of various TCM formulations or plant extracts in cancer patients, continued, systematic efforts in conducting scientifically sound studies with RCTs are required [144].

\section{Other Anticancer Applications of Specific Herbal Medicines}

Tumor microenvironments are now recognized to play a critical role in cancer growth, progression, and metastasis [145, 146]. Intensive interactions between tumor or cancerous cells and their stromal microenvironments that involve a spectrum of immune cell types have received considerable research attention over the past few years [146-148]. There has been particular interest in the strong link between various immune activities at or surrounding tumor tissues and the progression of tumor growth. Enhancement of tumor surveillance by the host immune system has also been considered to be a key strategy to facilitate anticancer effect. In this section, we address the specific effects of herbal medicines on the enhancement of host immunity and review their molecular targets in anticancer activities. 
4.1. Herbal Medicines as Adjuvant for Dendritic Cell-(DCBased Vaccines. By definition, the function of an adjuvant used with a vaccine is to aid or promote antigen delivery and presentation. An adjuvant can also assist in the induction of cytokines and stimulation/activation of antigen-presenting cells in the tumor or tissue microenvironment [149]. Specific herbal medicines such as Ganoderma lucidum or Dioscorea tuber have been reported to confer immunomodulatory activities [150, 151]. Bioactive polysaccharides from Ganoderma lucidum (Reishi) were investigated for their immunostimulatory and anticancer properties [152]. A specific polysaccharide fraction from Reishi stimulated immune cell activation including dendritic cell maturation and cytokine expression and displayed potent adjuvant activity in mice $[153,154]$. Polysaccharides from Dioscorea batatas were found to induce TNF- $\alpha$ secretion via Toll-like receptor 4-mediated protein kinase signaling pathways [155]. A number of phytochemicals have also been demonstrated to effectively enhance the anti-tumor potency of gene-based cancer vaccines. For example, shikonin enhanced the anti-tumor potency of a cancer vaccine via the induction of RANTES expression at the skin immunization tissue site [156]. And a phytocompound mixture extracted from the butanol fraction of a stem and leaf extract of Echinacea Purpurea conferred immunomodulatory effects suggesting that it can effectively modulate DC mobility and related cellular physiology in vivo in the mouse immune system [157]. These studies suggest the potential application of herbal medicines in a cell-based vaccine system.

4.2. Induction of Immunogenic Cell Death by Herbal Medicines. Immunogenic cell death mediated by damage-associated molecular pattern (DAMP) signals was found to trigger an immunogenic response including maturation and antigen uptake of dendritic cells [158]. Recently, Chen and colleagues [159] demonstrated that shikonin can induce immunogenic cell death in treated tumor cells. Shikonin-treated, tumor cell lysate-loaded, mature DCs were shown to exhibit strong anticancer activities against test mouse melanoma, including the induction of cytotoxic activities of splenocytes against target tumor cells, inhibition of tumor growth, and improvement in mouse survival. The use of shikonin-treated tumor cells from patients to pulse their own DCs in culture should be evaluated in future clinical studies as a new approach for developing DC-based anticancer vaccines.

\section{Conclusion and Future Development}

For centuries if not millennia, various plants (many systematized in traditional Chinese medicine) have been used as medicines and disease therapeutics in most human cultures. As exemplified in this review, over the last two decades renewed public interest and research efforts from scientific and medical communities worldwide have generated a large volume of information including clinical studies and trials on the pharmacological effects, usage, and the development into future medicines of herbs and derivative medicinal phytochemicals as anti-tumor and chemoprevention agents.
Although considerable effort has been put into the verification and upgrade of many traditional remedies or multipleherb formulations, systematic, standardized research and the use of FDA regulatory protocols and defined clinical trials are still quite limited and need to be actively pursued. At the same time, it is necessary for scientists, clinicians, and regulatory agencies to actively consider how to create novel, improved, or modified clinical surveys, studies, and trial mechanisms that employ the stringent trial standards of the 21st century but also incorporate, at the international level, the wealth of old empirical but incomplete data from various records and documents accumulated by traditional medicine practices worldwide, to expedite the discovery and development of new phytomedicines and botanical drugs.

While continuous and systematic effort is needed, a number of notable "breakthroughs" have occurred in the field of medicinal plant research and botanical drugs in the last few years. In April 2008, the FDA approved the very first botanical drug, Veregen, a partially purified fraction of the water extract of green tea leaves from Camellia sinensis, for topical treatment of external genital and perianal warts [160]. Very recently (January 2013), the FDA approved, for the first time, an oral botanical drug, Crofelemer (a purified oligomeric proanthocyanidin from the latex of the South American Croton lechleri tree), for treatment of diarrhea in HIV/AIDS patients. Although these two pioneer FDA-approved botanical drugs are not therapies for cancer, they certainly pave way for such future developments. One possible example is the ongoing (2013) FDA clinical trial on "PHY906." This fourherbal-plant-composed TCM formulation has been shown to confer with good evidence [74]. It is our hope that the phase III clinical trial of this formula will lead the way in the development of CAM for cancer patients. With the various other new clinical trials ongoing, CAM may start playing critical roles in future health care of aging populations.

\section{Conflict of Interests}

The authors declare no conflict of interests.

\section{Acknowledgments}

This work was supported by the Academia Sinica Investigator Award (2010-2014), Taiwan. The authors also thank Ms. Miranda Loney of Academia Sinica for paper editing.

\section{References}

[1] B. Gerber, H. Müller, T. Reimer, A. Krause, and K. Friese, "Nutrition and lifestyle factors on the risk of developing breast cancer," Breast Cancer Research and Treatment, vol. 79, no. 2, pp. 265-276, 2003.

[2] M. C. Shun, W. Yu, A. Gapor et al., "Pro-apoptotic mechanisms of action of a novel vitamin E analog ( $\alpha$-TEA) and a naturally occurring form of vitamin $\mathrm{E}$ ( $\delta$-Tocotrienol) in MDA-MB-435 human breast cancer cells," Nutrition and Cancer, vol. 48, no. 1 , pp. 95-105, 2004.

[3] X. F. Wang, P. K. Witting, B. A. Salvatore, and J. Neuzil, "Vitamin $\mathrm{E}$ analogs trigger apoptosis in HER2/erbB2-overexpressing 
breast cancer cells by signaling via the mitochondrial pathway," Biochemical and Biophysical Research Communications, vol. 326, no. 2, pp. 282-289, 2005.

[4] B. Gerber, C. Scholz, T. Reimer, V. Briese, and W. Janni, "Complementary and alternative therapeutic approaches in patients with early breast cancer: a systematic review," Breast Cancer Research and Treatment, vol. 95, no. 3, pp. 199-209, 2006.

[5] M. L. Lesperance, I. A. Olivotto, N. Forde et al., "Mega-dose vitamins and minerals in the treatment of non-metastatic breast cancer: an historical cohort study," Breast Cancer Research and Treatment, vol. 76, no. 2, pp. 137-143, 2002.

[6] M. Saintot, H. Mathieu-Daude, C. Astre, J. Grenier, J. SimonyLafontaine, and M. Gerber, "Oxidant-antioxidant status in relation to survival among breast cancer patients," International Journal of Cancer, vol. 97, no. 5, pp. 574-579, 2002.

[7] W. C. Willett and M. J. Stampfer, "What vitamins should I be taking, doctor?" The New England Journal of Medicine, vol. 345, no. 25, pp. 1819-1824, 2001.

[8] D. Ingram, K. Sanders, M. Kolybaba, and D. Lopez, "Casecontrol study of phyto-oestrogens and breast cancer," The Lancet, vol. 350, no. 9083, pp. 990-994, 1997.

[9] M. J. Messina, "Legumes and soybeans: overview of their nutritional profiles and health effects," The American Journal of Clinical Nutrition, vol. 70, no. 3, supplement, pp. 439S-450S, 1999.

[10] S. Yamamoto, T. Sobue, M. Kobayashi et al., "Soy, isoflavones, and breast cancer risk in Japan," Journal of the National Cancer Institute, vol. 95, no. 12, pp. 906-913, 2003.

[11] L. A. Fitzpatrick, "Phytoestrogens-mechanism of action and effect on bone markers and bone mineral density," Endocrinology and Metabolism Clinics of North America, vol. 32, no. 1, pp. 233-252, 2003.

[12] J. A. Tice, B. Ettinger, K. Ensrud, R. Wallace, T. Blackwell, and S. R. Cummings, "Phytoestrogen supplements for the treatment of hot flashes: the isoflavone clover extract (ICE) study: a randomized controlled trial," The Journal of the American Medical Association, vol. 290, no. 2, pp. 207-214, 2003.

[13] Society AC., "Breast cancer facts and figures," 2009.

[14] Society AC., "What are the key statistics about lung," 2013.

[15] K. J. Franek, Z. Zhou, W. D. Zhang, and W. Y. Chen, "In vitro studies of baicalin alone or in combination with Salvia miltiorrhiza extract as a potential anti-cancer agent," International Journal of Oncology, vol. 26, no. 1, pp. 217-224, 2005.

[16] W. Jonat, K. I. Pritchard, R. Sainsbury, and J. G. Klijn, “Trends in endocrine therapy and chemotherapy for early breast cancer: a focus on the premenopausal patient," Journal of Cancer Research and Clinical Oncology, vol. 132, no. 5, pp. 275-286, 2006.

[17] G. H. Di, H. C. Li, Z. Z. Shen, and Z. M. Shao, "Analysis of antiproliferation of curcumin on human breast cancer cells and its mechanism," Zhonghua Yi Xue Za Zhi, vol. 83, no. 20, pp. 17641768, 2003.

[18] C. Wu, F. Chen, J. W. Rushing et al., "Antiproliferative activities of parthenolide and golden feverfew extract against three human cancer cell lines," Journal of Medicinal Food, vol. 9, no. 1, pp. 55-61, 2006.

[19] W. Zhang, Y. Li, G. Zhang, J. Lü, and H. Ou, "Experimental study on MCF-7 cell apoptosis induced by ursolic acid," Zhong Yao Cai, vol. 28, no. 4, pp. 297-301, 2005.

[20] P. L. Kuo, Y. L. Hsu, and C. Y. Cho, "Plumbagin induces G2-M arrest and autophagy by inhibiting the AKT/mammalian target of rapamycin pathway in breast cancer cells," Molecular Cancer Therapeutics, vol. 5, no. 12, pp. 3209-3221, 2006.
[21] S. N. Sundar, C. N. Marconett, V. B. Doan, J. A. Willoughby Sr., and G. L. Firestone, "Artemisinin selectively decreases functional levels of estrogen receptor- $\alpha$ and ablates estrogeninduced proliferation in human breast cancer cells," Carcinogenesis, vol. 29, no. 12, pp. 2252-2258, 2008.

[22] P. S. Nelson and B. Montgomery, "Unconventional therapy for prostate cancer: good, bad or questionable?" Nature Reviews Cancer, vol. 3, no. 11, pp. 845-858, 2003.

[23] R. H. Fletcher and K. M. Fairfield, "Vitamins for chronic disease prevention in adults: clinical applications," The Journal of the American Medical Association, vol. 287, no. 23, pp. 3127-3129, 2002.

[24] L. A. Habel, W. Zhao, and J. L. Stanford, "Daily aspirin use and prostate cancer risk in a large, multiracial cohort in the US," Cancer Causes and Control, vol. 13, no. 5, pp. 427-434, 2002.

[25] R. B. Hayes, L. M. Pottern, H. Strickler et al., "Sexual behaviour, STDs and risks for prostate cancer," The British Journal of Cancer, vol. 82, no. 3, pp. 718-725, 2000.

[26] W. G. Nelson, A. M. de Marzo, and W. B. Isaacs, "Prostate cancer," The New England Journal of Medicine, vol. 349, no. 4, pp. 366-381, 2003.

[27] G. J. Kelloff, J. A. Crowell, V. E. Steele et al., "Progress in cancer chemoprevention: development of diet-derived chemopreventive agents," Journal of Nutrition, vol. 130, no. 2, supplement, pp. 467S-471S, 2000.

[28] H. A. Jones, J. M. Metz, P. Devine, S. M. Hahn, and R. Whittington, "Rates of unconventional medical therapy use in patients with prostate cancer: standard history versus directed questions," Urology, vol. 59, no. 2, pp. 272-276, 2002.

[29] R. K. Nam, N. Fleshner, E. Rakovitch et al., "Prevalence and patterns of the use of complementary therapies among prostate cancer patients: an epidemiological analysis," Journal of Urology, vol. 161, no. 5, pp. 1521-1524, 1999.

[30] J. M. Chan, M. J. Stampfer, J. Ma, E. B. Rimm, W. C. Willett, and E. L. Giovannucci, "Supplemental vitamin E intake and prostate cancer risk in a large cohort of men in the United States," Cancer Epidemiology Biomarkers and Prevention, vol. 8, no. 10, pp. 893899, 1999.

[31] E. A. Klein, I. M. Thompson, S. M. Lippman et al., "Select: the next prostate cancer prevention trial," Journal of Urology, vol. 166, no. 4, pp. 1311-1315, 2001.

[32] E. A. Klein, I. M. Thompson Jr., C. M. Tangen et al., "Vitamin $\mathrm{E}$ and the risk of prostate cancer: the selenium and vitamin $\mathrm{E}$ cancer prevention trial (SELECT)," The Journal of the American Medical Association, vol. 306, no. 14, pp. 1549-1556, 2011.

[33] D. N. Syed, N. Khan, F. Afaq, and H. Mukhtar, "Chemoprevention of prostate cancer through dietary agents: progress and promise," Cancer Epidemiology Biomarkers and Prevention, vol. 16, no. 11, pp. 2193-2203, 2007.

[34] S. Gupta, T. Hussain, and H. Mukhtar, "Molecular pathway for (-)-epigallocatechin-3-gallate-induced cell cycle arrest and apoptosis of human prostate carcinoma cells," Archives of Biochemistry and Biophysics, vol. 410, no. 1, pp. 177-185, 2003.

[35] S. Garbisa, S. Biggin, N. Cavallarin, L. Sartor, R. Benelli, and A. Albini, "Tumor invasion: molecular shears blunted by green tea," Nature Medicine, vol. 5, no. 11, p. 1216, 1999.

[36] A. Jatoi, N. Ellison, P. A. Burch et al., "A phase II trial of green tea in the treatment of patients with androgen independent metastatic prostate carcinoma," Cancer, vol. 97, no. 6, pp. 1442-1446, 2003. 
[37] B. A. J. Evans, K. Griffiths, and M. S. Morton, "Inhibition of $5 \alpha$-reductase in genital skin fibroblasts and prostate tissue by dietary lignans and isoflavonoids," Journal of Endocrinology, vol. 147, no. 2, pp. 295-302, 1995.

[38] S. Chen, Q. Ruan, E. Bedner et al., "Effects of the flavonoid baicalin and its metabolite baicalein on androgen receptor expression, cell cycle progression and apoptosis of prostate cancer cell lines," Cell Proliferation, vol. 34, no. 5, pp. 293-304, 2001.

[39] R. Miocinovic, N. P. McCabe, R. W. Keck, J. Jankun, J. A. Hampton, and S. H. Selman, "In vivo and in vitro effect of baicalein on human prostate cancer cells," International Journal of Oncology, vol. 26, no. 1, pp. 241-246, 2005.

[40] G. P. Pidgeon, M. Kandouz, A. Meram, and K. V. Honn, "Mechanisms controlling cell cycle arrest and induction of apoptosis after 12-lipoxygenase inhibition in prostate cancer cells," Cancer Research, vol. 62, no. 9, pp. 2721-2727, 2002.

[41] E. Giovannucci, E. B. Rimm, Y. Liu, M. J. Stampfer, and W. C. Willett, "A prospective study of tomato products, lycopene, and prostate cancer risk," Journal of the National Cancer Institute, vol. 94, no. 5, pp. 391-398, 2002.

[42] L. Chen, M. Stacewicz-Sapuntzakis, C. Duncan et al., "Oxidative DNA damage in prostate cancer patients consuming tomato sauce-based entrees as a whole-food intervention," Journal of the National Cancer Institute, vol. 93, no. 24, pp. 1872-1879, 2001.

[43] R. S. Dipaola, H. Zhang, G. H. Lambert et al., "Clinical and biologic activity of an estrogenic herbal combination (PC-SPES) in prostate cancer," The New England Journal of Medicine, vol. 339, no. 12, pp. 785-791, 1998.

[44] L. S. Marks, R. S. DiPaola, P. Nelson et al., "PC-SPES: herbal formulation for prostate cancer," Urology, vol. 60, no. 3, pp. 369375, 2002.

[45] H. S. Pandha and R. S. Kirby, "PC-SPES: phytotherapy for prostate cancer," The Lancet, vol. 359, no. 9325, pp. 2213-2215, 2002.

[46] F. M. Lin, L. R. Chen, E. H. Lin et al., "Compounds from Wedelia chinensis synergistically suppress androgen activity and growth in prostate cancer cells," Carcinogenesis, vol. 28, no. 12, pp. 25212529, 2007.

[47] C. H. Tsai, F. M. Lin, Y. C. Yang et al., "Herbal extract of Wedelia chinensis attenuates androgen receptor activity and orthotopic growth of prostate cancer in nude mice," Clinical Cancer Research, vol. 15, no. 17, pp. 5435-5444, 2009.

[48] Y. T. Huang, C. C. Wen, Y. H. Chen et al., "Dietary uptake of Wedelia chinensis extract attenuates dextran sulfate sodiuminduced colitis in mice," PLoS ONE, vol. 8, no. 5, Article ID e64152, 2013.

[49] L. E. Bröker and G. Giaccone, "The role of new agents in the treatment of non-small cell lung cancer," European Journal of Cancer, vol. 38, no. 18, pp. 2347-2361, 2002.

[50] G. Giaccone, "Targeted therapy in non-small cell lung cancer," Lung Cancer, vol. 38, supplement 2, pp. S29-S32, 2002.

[51] H. L. M. Liang, C. C. L. Xue, D. H. Zhou, and C. G. Li, "Chinese herbal medicine for lung cancer: a critical literature review," Chinese Journal of Integrated Medicine, vol. 9, pp. 157-160, 2011.

[52] M. A. Richardson and J. D. White, "Complementary/alternative medicine and cancer research: a national initiative," Cancer Practice, vol. 8, no. 1, pp. 45-48, 2000.

[53] I. Sakaida, M. Tsuchiya, K. Kawaguchi, T. Kimura, S. Terai, and K. Okita, "Herbal medicine Inchin-ko-to (TJ-135) prevents liver fibrosis and enzyme-altered lesions in rat liver cirrhosis induced by a choline-deficient L-amino acid-defined diet," Journal of Hepatology, vol. 38, no. 6, pp. 762-769, 2003.
[54] H. J. Lin, J. Y. Chen, C. F. Lin et al., "Hepatoprotective effects of Yi Guan Jian, an herbal medicine, in rats with dimethylnitrosamine-induced liver fibrosis," Journal of Ethnopharmacology, vol. 134, no. 3, pp. 953-960, 2011.

[55] X. Lin, S. Zhang, Q. Huang et al., "Protective effect of FufangLiu-Yue-Qing, a traditional Chinese herbal formula, on $\mathrm{CCl}_{4}$ induced liver fibrosis in rats," Journal of Ethnopharmacology, vol. 142, no. 2, pp. 548-556, 2012.

[56] J. Lv, Z. Zhao, Y. Chen et al., "The chinese herbal decoction danggui buxue tang inhibits angiogenesis in a rat model of liver fibrosis," Evidence-Based Complementary and Alternative Medicine, vol. 2012, Article ID 284963, 11 pages, 2012.

[57] J. M. Luk, X. Wang, P. Liu et al., “Traditional Chinese herbal medicines for treatment of liver fibrosis and cancer: from laboratory discovery to clinical evaluation," Liver International, vol. 27, no. 7, pp. 879-890, 2007.

[58] T. L. Wang, B.E. Wang, H. H. Zhang, X. Liu, Z. P. Duan, and J. Zhang, "Pathological study of the therapeutic effect on HBV-related liver fibrosis with herbal compound 861," Chinese Journal of Gastroenterology and Hepatology, vol. 7, pp. 148-153, 1998.

[59] H. J. Wang and B. E. W. Wang, "Long-term follow-up result of compound Dan Shen granule (861 Chong Fu Ji) in treating hepatofibrosis," Chinese Journal of Integrated Traditional and Western Medicine, vol. 5, pp. 4-5, 1995.

[60] H. Oka, S. Yamamoto, T. Kuroki et al., "Prospective study of chemoprevention of hepatocellular carcinoma with Sho-saikoto (TJ-9)," Cancer, vol. 76, no. 5, pp. 743-749, 1995.

[61] G. J. P. Dijkgraaf, B. Alicke, L. Weinmann et al., "Small molecule inhibition of GDC-0449 refractory smoothened mutants and downstream mechanisms of drug resistance," Cancer Research, vol. 71, no. 2, pp. 435-444, 2011.

[62] J. K. Chen, J. Taipale, M. K. Cooper, and P. A. Beachy, "Inhibition of Hedgehog signaling by direct binding of cyclopamine to Smoothened," Genes and Development, vol. 16, no. 21, pp. 27432748, 2002.

[63] G. Feldmann, S. Dhara, V. Fendrich et al., "Blockade of hedgehog signaling inhibits pancreatic cancer invasion and metastases: a new paradigm for combination therapy in solid cancers," Cancer Research, vol. 67, no. 5, pp. 2187-2196, 2007.

[64] S. Ko, H. Kanehiro, M. Hisanaga, M. Nagao, N. Ikeda, and Y. Nakajima, "Liver fibrosis increases the risk of intrahepatic recurrence after hepatectomy for hepatocellular carcinoma," The British Journal of Surgery, vol. 89, no. 1, pp. 57-62, 2002.

[65] H. Nakayama, T. Takayama, and A. Hemmi, "Postoperative complications of hepatectomy for hepatocellular carcinoma in relation to liver fibrosis," Hepato-Gastroenterology, vol. 52, no. 62, pp. 587-590, 2005.

[66] K. Kayano, I. Sakaida, K. Uchida, and K. Okita, "Inhibitory effects of the herbal medicine Sho-saiko-to (TJ-9) on cell proliferation and procollagen gene expressions in cultured rat hepatic stellate cells," Journal of Hepatology, vol. 29, no. 4, pp. 642-649, 1998.

[67] I. Sakaida, Y. Matsumura, S. Akiyama, K. Hayashi, A. Ishige, and K. Okita, "Herbal medicine Sho-saiko-to (TJ-9) prevents liver fibrosis and enzyme- altered lesions in rat liver cirrhosis induced by a choline-deficient L-amino acid-defined diet," Journal of Hepatology, vol. 28, no. 2, pp. 298-306, 1998.

[68] J. K. Lee, J. H. Kim, and H. K. Shin, "Therapeutic effects of the oriental herbal medicine Sho-saiko-to on liver cirrhosis and carcinoma," Hepatology Research, vol. 41, no. 9, pp. 825-837, 2011. 
[69] F. Stickel and D. Schuppan, "Herbal medicine in the treatment of liver diseases," Digestive and Liver Disease, vol. 39, no. 4, pp. 293-304, 2007.

[70] D. M. Simeone, "Pancreatic cancer stem cells: implications for the treatment of pancreatic cancer," Clinical Cancer Research, vol. 14 , no. 18 , pp. 5646-5648, 2008.

[71] J. P. Morton, M. E. Mongeau, D. S. Klimstra et al., "Sonic hedgehog acts at multiple stages during pancreatic tumorigenesis," Proceedings of the National Academy of Sciences of the United States of America, vol. 104, no. 12, pp. 5103-5108, 2007.

[72] A. S. M. Abdullah, Y. Lau, and L. W. C. Chow, "Pattern of alternative medicine usage among the Chinese breast cancer patients: implication for service integration," The American Journal of Chinese Medicine, vol. 31, no. 4, pp. 649-658, 2003.

[73] Y. Cui, X. O. Shu, Y. Gao et al., "Use of complementary and alternative medicine by Chinese women with breast cancer," Breast Cancer Research and Treatment, vol. 85, no. 3, pp. 263270, 2004.

[74] W. Lam, S. Bussom, F. Guan et al., "Chemotherapy: the four-herb Chinese medicine PHY906 reduces chemotherapyinduced gastrointestinal toxicity," Science Translational Medicine, vol. 2, no. 45, Article ID 45ra59, 2010.

[75] M. A. Ferro, A. Leis, R. Doll, L. Chiu, M. Chung, and M. Barroetavena, "The impact of acculturation on the use of traditional Chinese medicine in newly diagnosed Chinese cancer patients," Supportive Care in Cancer, vol. 15, no. 8, pp. 985-992, 2007.

[76] G. Maskarinec, D. M. Shumay, H. Kakai, and C. C. Gotay, "Ethnic differences in complementary and alternative medicine use among cancer patients," Journal of Alternative and Complementary Medicine, vol. 6, no. 6, pp. 531-538, 2000.

[77] F. Qi, A. Li, Y. Inagaki et al., "Chinese herbal medicines as adjuvant treatment during chemo- or radio-therapy for cancer," Bioscience Trends, vol. 4, no. 6, pp. 297-307, 2010.

[78] Y. Yoshida, M. Q. Wang, J. N. Liu, B. E. Shan, and U. Yamashita, "Immunomodulating activity of Chinese medicinal herbs and Oldenlandia diffusa in particular," International Journal of Immunopharmacology, vol. 19, no. 7, pp. 359-370, 1997.

[79] Y. Wang, X. J. Qian, H. R. Hadley, and B. H. Lau, "Phytochemicals potentiate interleukin-2 generated lymphokine-activated killer cell cytotoxicity against murine renal cell carcinoma," Molecular Biotherapy, vol. 4, no. 3, pp. 143-146, 1992.

[80] J. S. Jurenka, "Anti-inflammatory properties of curcumin, a major constituent of Curcuma longa: a review of preclinical and clinical research," Alternative Medicine Review, vol. 14, no. 2, pp. 141-153, 2009.

[81] H. Hatcher, R. Planalp, J. Cho, F. M. Torti, and S. V. Torti, "Curcumin: from ancient medicine to current clinical trials," Cellular and Molecular Life Sciences, vol. 65, no. 11, pp. 1631-1652, 2008.

[82] M. Li, Z. Zhang, D. L. Hill, H. Wang, and R. Zhang, "Curcumin, a dietary component, has anticancer, chemosensitization, and radiosensitization effects by down-regulating the MDM2 oncogene through the PI3K/mTOR/ETS2 pathway," Cancer Research, vol. 67, no. 5, pp. 1988-1996, 2007.

[83] L. J. Hofseth and M. J. Wargovich, "Inflammation, cancer, and targets of ginseng," Journal of Nutrition, vol. 137, no. 1, supplement, pp. 183S-185S, 2007.

[84] T. K. Lee, R. M. Johnke, R. R. Allison, K. F. O’Brien, and L. J. Dobbs Jr., "Radioprotective potential of ginseng," Mutagenesis, vol. 20, no. 4, pp. 237-243, 2005.
[85] T. K. Yun and S. Y. Choi, "Preventive effect of ginseng intake against various human cancers: a case-control study on 1987 pairs," Cancer Epidemiology Biomarkers and Prevention, vol. 4, no. 4, pp. 401-408, 1995.

[86] S. H. Kim, S. E. Lee, H. Oh et al., "The radioprotective effects of Bu-Zhong-Yi-Qi-Tang: a prescription of traditional Chinese medicine," The American Journal of Chinese Medicine, vol. 30, no. 1, pp. 127-137, 2002.

[87] J. S. Jeong, B. H. Ryu, J. S. Kim, J. W. Park, W. C. Choi, and S. W. Yoon, "Bojungikki-tang for cancer-related fatigue: a pilot randomized clinical trial," Integrative Cancer Therapies, vol. 9, no. 4, pp. 331-338, 2010.

[88] M. W. Saif, F. Lansigan, S. Ruta et al., "Phase I study of the botanical formulation PHY906 with capecitabine in advanced pancreatic and other gastrointestinal malignancies," Phytomedicine, vol. 17, no. 3-4, pp. 161-169, 2010.

[89] Y. Yen, S. So, M. Rose et al., "Phase I/II study of PHY906/ capecitabine in advanced hepatocellular carcinoma," Anticancer Research, vol. 29, no. 10, pp. 4083-4092, 2009.

[90] M. P. Farrell and S. Kummar, "Phase I/IIA randomized study of PHY906, a novel herbal agent, as a modulator of chemotherapy in patients with advanced colorectal cancer," Clinical Colorectal Cancer, vol. 2, no. 4, pp. 253-256, 2003.

[91] Z. Meng, P. Yang, Y. Shen et al., "Pilot study of huachansu in patients with hepatocellular carcinoma, nonsmall-cell lung cancer, or pancreatic cancer," Cancer, vol. 115, no. 22, pp. 5309-5318, 2009.

[92] T. J. Qin, X. H. Zhao, J. Yun, L. Zhang, Z. Ruan, and B. Pan, "Efficacy and safety of gemcitabine-oxaliplatin combined with huachansu in patients with advanced gallbladder carcinoma," World Journal of Gastroenterology, vol. 14, no. 33, pp. 5210-5216, 2008.

[93] M. W. Wheeler and A. J. Bailer, "A simulation study of methods for constructing confidence intervals for bioaccumulation factors," Environmental Toxicology and Chemistry, vol. 22, no. 4, pp. 921-927, 2003.

[94] H. Y. Guo, Y. Cai, X. M. Yang et al., "Randomized phase II trial on mitomycin-C/Cisplatin \pm KLT in heavily pretreated advanced breast cancer," The American Journal of Chinese Medicine, vol. 36, no. 4, pp. 665-674, 2008.

[95] L. Xu, X. L. Li, A. Ge, S. Yu, J. Li, and P. J. Mansky, "Chinese herbal medicine for cancer pain," Integrative Cancer Therapies, vol. 6, no. 3, pp. 208-234, 2007.

[96] M. L. Liu, L. Y. Chien, C. J. Tai, K. C. Lin, and C. J. Tai, "Effectiveness of traditional Chinese medicine for liver protection and chemotherapy completion among cancer patients," Evidence-Based Complementary and Alternative Medicine, vol. 2011, Article ID 291843, 8 pages, 2011.

[97] Y. Tu, “The discovery of artemisinin (qinghaosu) and gifts from Chinese medicine," Nature Medicine, vol. 17, no. 10, pp. 12171220, 2011.

[98] Y. Z. Chen, Z. D. Li, F. Gao, Y. Zhang, H. Sun, and P. Li, "Effects of combined Chinese drugs and chemotherapy in treating advanced non-small cell lung cancer," Chinese Journal of Integrative Medicine, vol. 15, no. 6, pp. 415-419, 2009.

[99] H. S. Lin and D. R. Li, "Multi-center randomized clinical study on Shenqi-fuzheng injection combined with chemotherapy in the treatment for lung cancer," Zhonghua Zhong Liu Za Zhi, vol. 29, no. 12, pp. 931-934, 2007.

[100] J. You, Z. M. Shi, and B. H. Han, "Evaluation on effect of feiji recipe on quality of life of patients with non-small cell lung 
cancer by adopting international questionnaire of QOL," Zhongguo Zhong Xi Yi Jie He Za Zhi, vol. 26, no. 1, pp. 33-37, 2006.

[101] J. H. Tian, L. S. Liu, Z. M. Shi, Z. Y. Zhou, and L. Wang, "A randomized controlled pilot trial of "feiji recipe" on quality of life of non-small cell lung cancer patients," The American Journal of Chinese Medicine, vol. 38, no. 1, pp. 15-25, 2010.

[102] L. Xu, H. Li, Z. Xu et al., "Multi-center randomized doubleblind controlled clinical study of chemotherapy combined with or without traditional Chinese medicine on quality of life of postoperative non-small cell lung cancer patients," BMC Complementary and Alternative Medicine, vol. 12, article 112, 2012.

[103] B. Movsas, T. A. Raffin, A. H. Epstein, and C. J. Link Jr., "Pulmonary radiation injury," Chest, vol. 111, no. 4, pp. 1061-1076, 1997.

[104] Y. Q. Dou, M. H. Yang, Z. M. Wei, C. D. Xiao, and X. H. Yang, "The study of early application with Dixiong decoction for nonsmall cell lung cancer to decrease the incidence and severity of radiation pneumonitis: a prospective, randomized clinical trial," Chinese Journal of Integrative Medicine, vol. 16, no. 5, pp. 411-416, 2010.

[105] C. Xiao, H. J. Ding, L. C. Feng, B. L. Qu, and Y. Q. Dou, "Efficacy of Liangxue Jiedu Huoxue decoction in prevention of radiation pneumonitis: a randomized controlled trial," Journal of Chinese Integrative Medicine, vol. 8, no. 7, pp. 624-628, 2010.

[106] T. Zhang, S. L. Ma, and J. H. Yue, "Clinical observation on treatment of radiation pneumonia by Qingjin Runfei decoction combined with hormone and antibiotic," Zhongguo Zhong Xi Yi Jie He Za Zhi, vol. 27, no. 3, pp. 254-256, 2007.

[107] W. Zheng, Z. H. Gao, and L. N. Wu, "Clinical observation on treatment of radiative pneumonia in patients with lung cancer by integrative Chinese and Western medicine," Zhongguo Zhong Xi Yi Jie He Za Zhi, vol. 27, no. 12, pp. 1121-1123, 2007.

[108] S. J. Jeong, W. Koh, B. Kim, and S. H. Kim, "Are there new therapeutic options for treating lung cancer based on herbal medicines and their metabolites?" Journal of Ethnopharmacology, vol. 138, no. 3, pp. 652-661, 2011.

[109] C. D. Scripture and W. D. Figg, "Drug interactions in cancer therapy," Nature Reviews Cancer, vol. 6, no. 7, pp. 546-558, 2006.

[110] Y. Zhang and L. Z. Benet, "The gut as a barrier to drug absorption: combined role of cytochrome P450 3A and P-glycoprotein," Clinical Pharmacokinetics, vol. 40, no. 3, pp. 159-168, 2001.

[111] A. Sparreboom, M. C. Cox, M. R. Acharya, and W. D. Figg, "Herbal remedies in the United States: potential adverse interactions with anticancer agents," Journal of Clinical Oncology, vol. 22, no. 12, pp. 2489-2503, 2004.

[112] E. Ernst and B. R. Cassileth, "The prevalence of complementary/ alternative medicine in cancer: a systematic review," Cancer, vol. 83, no. 4, pp. 777-782, 1998.

[113] D. Dürr, B. Stieger, G. A. Kullak-Ublick et al., "St John's Wort induces intestinal P-glycoprotein/MDR1 and intestinal and hepatic CYP3A4," Clinical Pharmacology and Therapeutics, vol. 68, no. 6, pp. 598-604, 2000.

[114] R. H. J. Mathijssen, J. Verweij, P. de Bruijn, W. J. Loos, and A. Sparreboom, "Effects of St. John's Wort on irinotecan metabolism," Journal of the National Cancer Institute, vol. 94, no. 16, pp. 1247-1249, 2002.

[115] K. K. Auyeung and J. K. Ko, "Novel herbal flavonoids promote apoptosis but differentially induce cell cycle arrest in human colon cancer cell," Investigational New Drugs, vol. 28, no. 1, pp. $1-13,2010$.
[116] K. K. Au-Yeung, P. L. Liu, C. Chan, W. Wu, S. S. Lee, and J. K. Ko, "Herbal isoprenols induce apoptosis in human colon cancer cells through transcriptional activation of PPAR $\gamma$," Cancer Investigation, vol. 26, no. 7, pp. 708-717, 2008.

[117] J. P. A. A. Baak, C. Gyllenhaal, L. Liu, H. Guo, and K. I. Block, "Prognostic proof and possible therapeutic mechanisms of herbal medicine in patients with metastatic lung and colon cancer," Integrative Cancer Therapies, vol. 10, no. 3, pp. NP1NP11, 2011.

[118] M. McCulloch, M. Broffman, M. van der Laan et al., "Colon cancer survival with herbal medicine and vitamins combined with standard therapy in a whole-systems approach: ten-year follow-up data analyzed with marginal structural models and propensity score methods," Integrative Cancer Therapies, vol. 10, no. 3, pp. 240-259, 2011.

[119] S. Deng, B. Hu, and H. M. An, "Traditional chinese medicinal syndromes and treatment in colorectal cancer," Journal of Cancer Therapy, vol. 3, no. 6, pp. 888-897, 2012.

[120] W. Jiang, "Therapeutic wisdom in traditional Chinese medicine: a perspective from modern science," Trends in Pharmacological Sciences, vol. 26, no. 11, pp. 558-563, 2005.

[121] L. X. Ju, Z. Chen, and R. Z. Ren, "Progress in research on the treatment of primary liver cancer with traditional Chinese medicine for activating blood to resolve stasis," Zhong Xi Yi Jie He Xue Bao, vol. 3, no. 6, pp. 491-494, 2005.

[122] Q. Liu, Y. B. Zhang, C. H. Ma, X. Q. Yue, and C. Q. Ling, "Analysis of literature on therapeutic methods and medicines of traditional Chinese medicine for primary liver cancer," Zhong Xi Yi Jie He Xue Bao, vol. 3, no. 4, pp. 260-262, 2005.

[123] L. Z. Lin, D. H. Zhou, K. Liu, F. Wang, S. Lan, and X. Ye, "Analysis on the prognostic factors in patients with large hepatocarcinoma treated by shentao ruangan pill and hydroxycamptothecine," Zhongguo Zhong Xi Yi Jie He Za Zhi, vol. 25, no. 1, pp. 8-11, 2005.

[124] M. B. Meng, Y. L. Cui, Y. S. Guan et al., “Traditional Chinese medicine plus transcatheter arterial chemoembolization for unresectable hepatocellular carcinoma," Journal of Alternative and Complementary Medicine, vol. 14, no. 8, pp. 1027-1042, 2008.

[125] V. B. Konkimalla and T. Efferth, "Evidence-based Chinese medicine for cancer therapy," Journal of Ethnopharmacology, vol. 116, no. 2, pp. 207-210, 2008.

[126] W. C. S. Cho and H. Y. Chen, "Clinical efficacy of traditional chinese medicine as a concomitant therapy for nasopharyngeal carcinoma: a systematic review and meta-analysis," Cancer Investigation, vol. 27, no. 3, pp. 334-344, 2009.

[127] X. C. Su, Y. L. Wang, X. Y. Yang et al., "Effect of Guliu capsule combined with 89Sr therapy on metastatic bone tumor," $D i \mathrm{Yi}$ Jun Yi Da Xue Xue Bao, vol. 25, no. 9, pp. 1164-1177, 2005.

[128] D. Quan, Y. Lu, and Z. Li, "Clinical observation on radio- or chemotherapy plus traditional Chinese medicine in treating brain metastatic tumor," Zhongguo Zhong Xi Yi Jie He Za Zhi, vol. 19, no. 7, pp. 392-394, 1999.

[129] N. S. Yang, L. F. Shyur, C. H. Chen, S. Y. Wang, and C. M. Tzeng, "Medicinal herb extract and a single-compound drug confer similar complex pharmacogenomic activities in MCF-7 cells," Journal of Biomedical Science, vol. 11, no. 3, pp. 418-422, 2004.

[130] S. Y. Yin and N. S. Yang, "Immuno-modulatory effects of phytomedicines evaluated using omics approaches," Systems and Computational Biology-Molecular and Cellular Experimental Systems, no. 16, pp. 289-314, 2011. 
[131] P. Goldman, "Herbal medicines today and the roots of modern pharmacology," Annals of Internal Medicine, vol. 135, no. 8, pp. 594-600, 2001.

[132] Y. Z. Liang, P. Xie, and K. Chan, "Quality control of herbal medicines," Journal of Chromatography B, vol. 812, no. 1-2, pp. 53-70, 2004.

[133] W. J. Kong, Y. L. Zhao, X. H. Xiao, C. Jin, and Z. L. Li, "Quantitative and chemical fingerprint analysis for quality control of rhizoma Coptidischinensis based on UPLC-PAD combined with chemometrics methods," Phytomedicine, vol. 16, no. 10, pp. 950-959, 2009.

[134] Z. Yongyu, S. Shujun, D. Jianye et al., "Quality control method for herbal medicine-chemical fingerprint analysis," in Quality Control of Herbal Medicines and Related Area, pp. 171-194, 2011.

[135] L. You, R. An, K. Liang, and X. Wang, "Anti-breast cancer agents from Chinese herbal medicines," Mini-Reviews in Medicinal Chemistry, vol. 13, no. 1, pp. 101-105, 2013.

[136] D. G. Altman, K. F. Schulz, D. Moher et al., "The revised CONSORT statement for reporting randomized trials: explanation and elaboration," Annals of Internal Medicine, vol. 134, no. 8, pp. 663-694, 2001.

[137] A. R. Jadad, R. A. Moore, D. Carroll et al., "Assessing the quality of reports of randomized clinical trials: Is blinding necessary?" Controlled Clinical Trials, vol. 17, no. 1, pp. 1-12, 1996.

[138] H. MacPherson, A. White, M. Cummings, K. Jobst, K. Rose, and R. Niemtzow, "Standards for reporting interventions in controlled trials of acupuncture: the STRICTA recommendations," Complementary Therapies in Medicine, vol. 9, no. 4, pp. 246-249, 2001.

[139] K. Linde, W. B. Jonas, D. Melchart, and S. Willich, "The methodological quality of randomized controlled trials of homeopathy herbal medicines and acupuncture," International Journal of Epidemiology, vol. 30, no. 3, pp. 526-531, 2001.

[140] J. J. Gagnier, J. DeMelo, H. Boon, P. Rochon, and C. Bombardier, "Quality of reporting of randomized controlled trials of herbal medicine interventions," The American Journal of Medicine, vol. 119, no. 9, pp. 800.el-800.el1, 2006.

[141] J. J. Gagnier, H. Boon, P. Rochon, D. Moher, J. Barnes, and C. Bombardier, "Reporting randomized, controlled trials of herbal interventions: an elaborated CONSORT statement," Annals of Internal Medicine, vol. 144, no. 5, pp. 364-367, 2006.

[142] J. J. Gagnier, H. Boon, P. Rochon, D. Moher, J. Barnes, and C. Bombardier, "Recommendations for reporting randomized controlled trials of herbal interventions: explanation and elaboration," Journal of Clinical Epidemiology, vol. 59, no. 11, pp. 11341149, 2006.

[143] T. S. K. Mok, W. Yeo, P. J. Johnson et al., "A doubleblind placebo-controlled randomized study of Chinese herbal medicine as complementary therapy for reduction of chemotherapy-induced toxicity," Annals of Oncology, vol. 18, no. 4, pp. 768-774, 2007.

[144] K. K. L. Chan, T. J. Yao, B. Jones et al., "The use of Chinese herbal medicine to improve quality of life in women undergoing chemotherapy for ovarian cancer: a double-blind placebocontrolled randomized trial with immunological monitoring," Annals of Oncology, vol. 22, no. 10, pp. 2241-2249, 2011.

[145] A. Albini and M. B. Sporn, "The tumour microenvironment as a target for chemoprevention," Nature Reviews Cancer, vol. 7, no. 2, pp. 139-147, 2007.

[146] F. R. Balkwill, M. Capasso, and T. Hagemann, "The tumor microenvironment at a glance," Journal of Cell Science, vol. 125, part 23, pp. 5591-5596, 2012.
[147] A. Mantovani, P. Allavena, A. Sica, and F. Balkwill, "Cancerrelated inflammation," Nature, vol. 454, no. 7203, pp. 436-444, 2008.

[148] J. Marx, "Inflammation and cancer: the link grows stronger," Science, vol. 306, no. 5698, pp. 966-968, 2004.

[149] H. F. Staats, C. P. Bradney, W. M. Gwinn et al., "Cytokine requirements for induction of systemic and mucosal CTL after nasal immunization," The Journal of Immunology, vol. 167, no. 9, pp. 5386-5394, 2001.

[150] P. F. Su, C. J. Li, C. C. Hsu et al., "Dioscorea phytocompounds enhance murine splenocyte proliferation ex vivo and improve regeneration of bone marrow cells in vivo," Evidence-Based Complementary and Alternative Medicine, vol. 2011, Article ID 731308, 11 pages, 2011.

[151] H. Y. Hsu, K. F. Hua, C. C. Lin, C. Lin, J. Hsu, and C. Wong, "Extract of Reishi polysaccharides induces cytokine expression via TLR4-modulated protein kinase signaling pathways," Journal of Immunology, vol. 173, no. 10, pp. 5989-5999, 2004.

[152] R. Chang, "Bioactive polysaccharides from traditional chinese medicine herbs as anticancer adjuvants," Journal of Alternative and Complementary Medicine, vol. 8, no. 5, pp. 559-565, 2002.

[153] H. S. Chen, Y. F. Tsai, S. Lin et al., "Studies on the immunomodulating and anti-tumor activities of Ganoderma lucidum (Reishi) polysaccharides," Bioorganic and Medicinal Chemistry, vol. 12, no. 21, pp. 5595-5601, 2004.

[154] C. Y. Lai, J. T. Hung, H. H. Lin et al., "Immunomodulatory and adjuvant activities of a polysaccharide extract of Ganoderma lucidum in vivo and in vitro," Vaccine, vol. 28, no. 31, pp. 49454954, 2010.

[155] J. Y. Liu, F. L. Yang, C. P. Lu et al., "Polysaccharides from Dioscorea batatas induce tumor necrosis factor- $\alpha$ secretion via toll-like receptor 4-mediated protein kinase signaling pathways," Journal of Agricultural and Food Chemistry, vol. 56, no. 21, pp. 9892-9898, 2008.

[156] H. M. Chen, P. H. Wang, K. Aravindaram et al., "Shikonin enhances efficacy of a gene-based cancer vaccine via induction of RANTES," Journal of Biomedical Science, vol. 19, article 42, 2012.

[157] S. Y. Yin, W. H. Wang, P. H. Wang et al., "Stimulatory effect of Echinacea purpurea extract on the trafficking activity of mouse dendritic cells: revealed by genomic and proteomic analyses," BMC Genomics, vol. 11, no. 1, article 612, 2010.

[158] D. V. Krysko, A. D. Garg, A. Kaczmarek, O. Krysko, P. Agostinis, and P. Vandenabeele, "Immunogenic cell death and DAMPs in cancer therapy," Nature Reviews Cancer, vol. 12, no. 12, pp. 860$875,2012$.

[159] H. M. Chen, P. H. Wang, S. S. Chen et al., "Shikonin induces immunogenic cell death in tumor cells and enhances dendritic cell-based cancer vaccine," Cancer Immunology, Immunotherapy, vol. 61, no. 11, pp. 1989-2002, 2012.

[160] K. M. Wu, H. Ghantous, and D. B. Birnkrant, "Current regulatory toxicology perspectives on the development of herbal medicines to prescription drug products in the United States," Food and Chemical Toxicology, vol. 46, no. 8, pp. 2606-2610, 2008. 


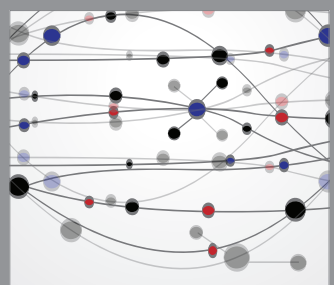

The Scientific World Journal
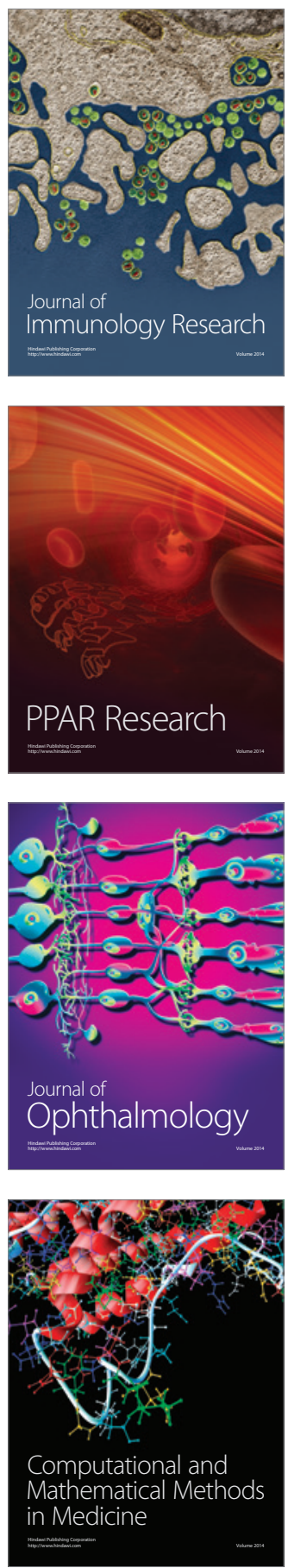

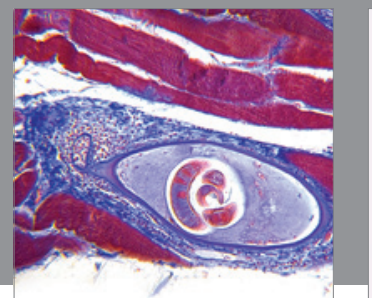

Gastroenterology

Research and Practice
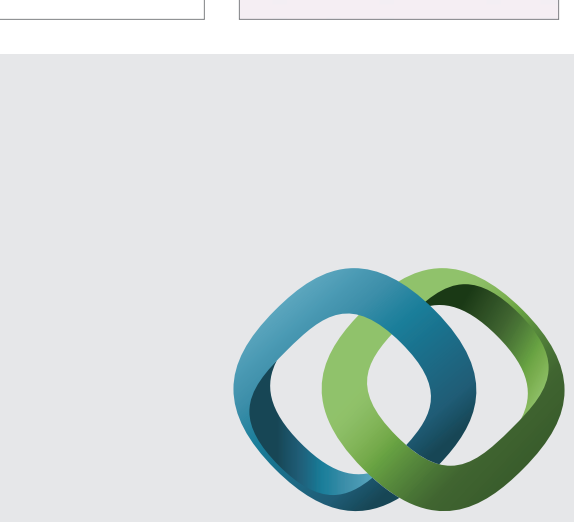

\section{Hindawi}

Submit your manuscripts at

http://www.hindawi.com
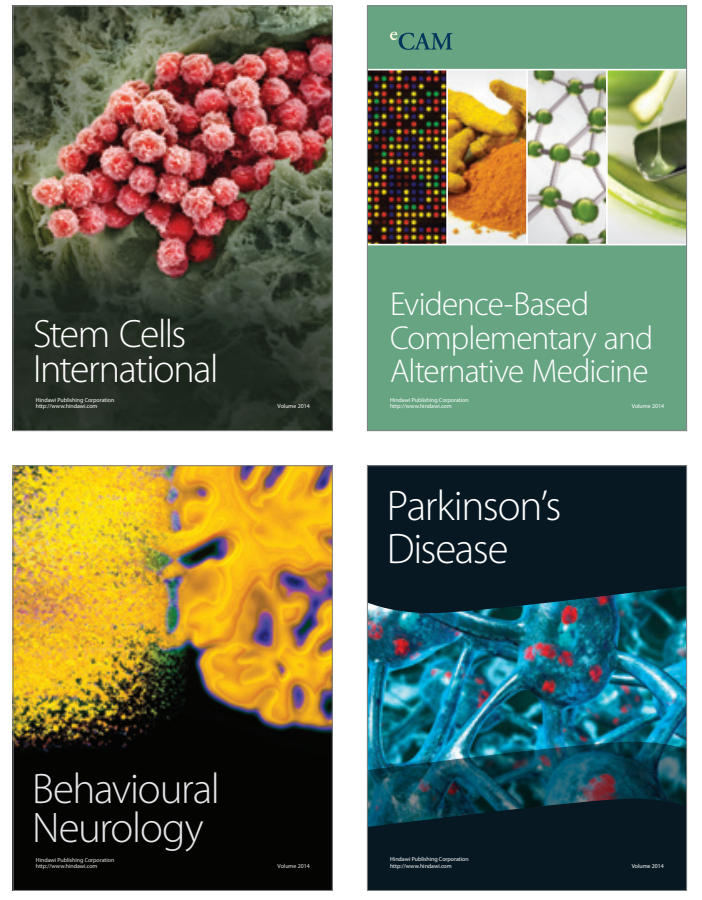
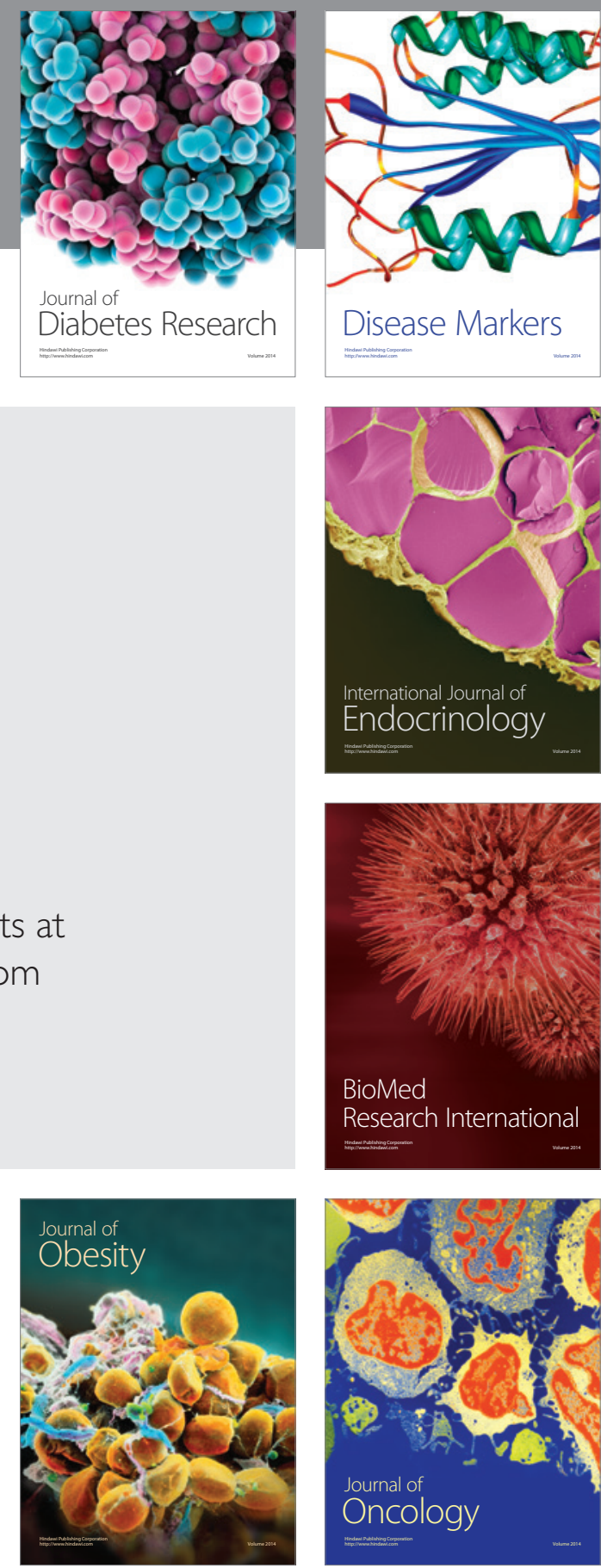

Disease Markers
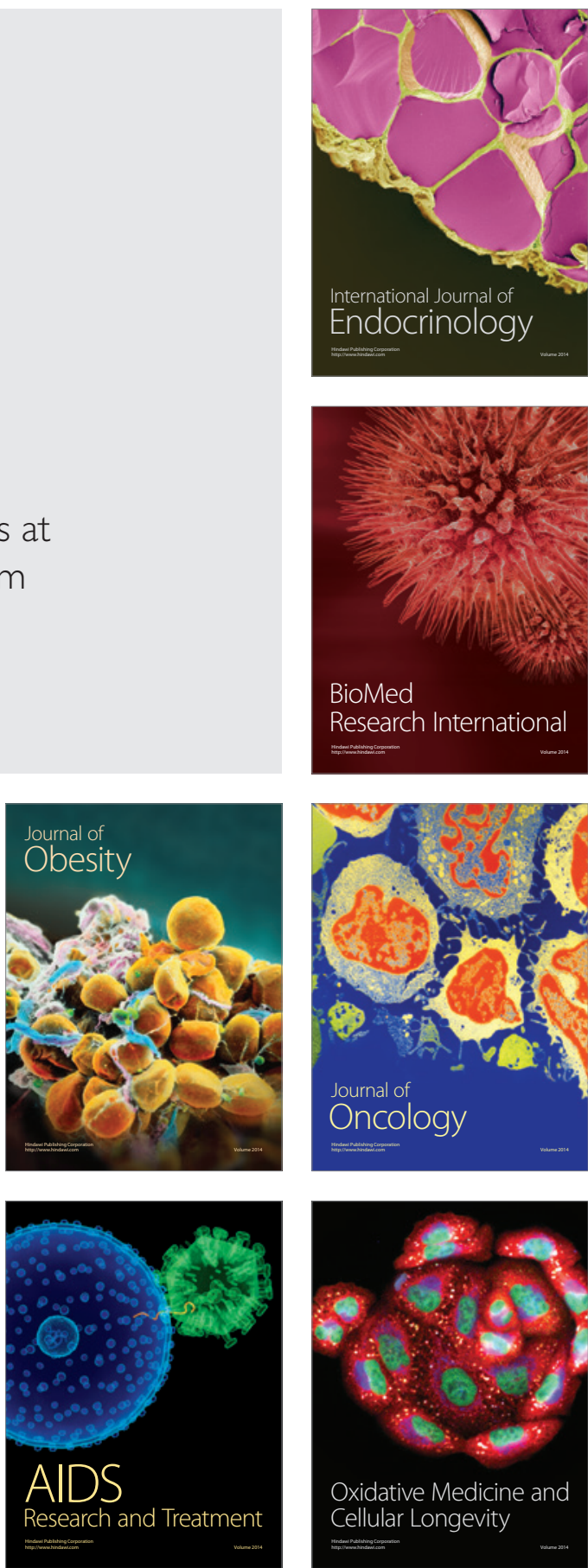